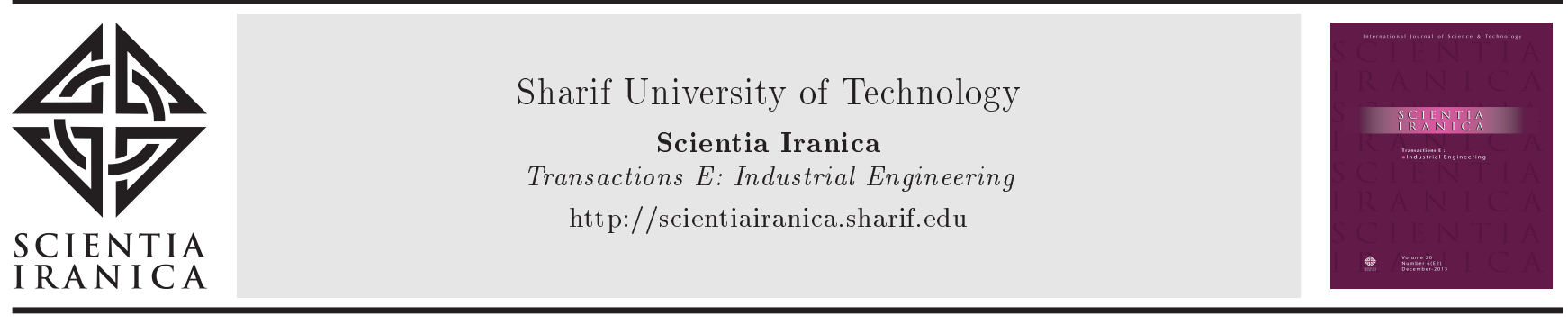

\title{
Designing a resource-constrained project scheduling model considering multiple routes for flexible project activities: Meta-heuristic algorithms
}

\author{
A. Birjandi ${ }^{a}$, S.M. Mousavi ${ }^{\mathrm{b}, *}$, and B. Vahdani ${ }^{\mathrm{c}}$ \\ a. Department of Industrial Engineering, South Tehran Branch, Islamic Azad University, Tehran, Iran. \\ b. Department of Industrial Engineering, Shahed University, Tehran, Iran. \\ c. Department of Industrial Engineering, Faculty of Industrial and Mechanical Engineering, Qazvin Branch, Islamic Azad \\ University, Qazvin, Iran.
}

Received 13 April 2018; received in revised form 30 October 2018; accepted 16 February 2019

\author{
KEYWORDS \\ Resource-Constrained \\ Project Scheduling \\ Problem (RCPSP); \\ Flexible activities; \\ Multiple routes; \\ Particle Swarm \\ Optimization (PSO); \\ Genetic Algorithm \\ (GA).
}

\begin{abstract}
Resource-Constrained Project Scheduling Problem with Multiple Routes (RCPSP-MR) for flexible project activities is a generalization of the RCPSP in which, for the implementation of each flexible activity in the main structure of the project, several exclusive sub-networks are considered. Each sub-network is regarded as a route for flexible activity. The routes considered for each flexible activity are varied in terms of: 1) the number of activities requiring to be executed; 2) the precedence relationships between activates; 3) allocation of different renewable and nonrenewable resources to each activity; and 4) effectiveness in duration and cost of project completion. In this study, first, a new mathematical formulation for RCPSP-MR was developed. Then, two solving approaches based on Particle Swarm Optimization (PSO) and Genetic Algorithm (GA) were adopted to minimize the costs of project completion. To evaluate the effectiveness of the proposed approaches, 50 problems (in very small, small, medium, and large-size test problems) were designed and solved. Finally, comparisons were made between the results. Computational results showed that the proposed GA would generate high-quality solutions in a timely fashion.
\end{abstract}

(C) 2020 Sharif University of Technology. All rights reserved.

\section{Introduction}

Project Scheduling Problem (PSP) determines run times for a specific set of fixed activities with regard to the precedent relationships via the allocation of different resources in order to achieve predetermined goals [1-4]. The role of the PSP and baseline scheduling in project management is crucial [5-8] and for this reason, Vanhoucke [9] presented nine time and eight cost forecasting methods for both project duration and

*. Corresponding author. Tel./Fax: +982151212091 E-mail address: sm.mousavi@shahed.ac.ir (S.M. Mousavi)

doi: $10.24200 /$ sci.2019.50778.1860 cost. Project scheduling has attracted considerable attention because of its critical role in resource management when Resource-Constrained Project Scheduling Problem (RCPSP) is turned into a standard problem. RCPSP from the classical viewpoint is defined as scheduling a set of fixed activities with precedence constraints implemented under limited availability of resources to minimize the makespan $[10,11]$. RCPSP is a basic scheduling problem which comprises activities, scarce resources to execute activities, and precedence relations between activities. The goal is to find an optimal schedule satisfying the resource and precedence constraints. These scheduling problems have many applications, ranging from production planning to project management [12]. Accordingly, RCPSP 
determines a proper sequence of activities in which two main constraints are involved: (1) resource constraints and (2) precedence relationships. These constraints are satisfied at fashion time and measurement criteria, such as time, cost, and quality, are optimized [1]. The classification in RCPSP is summarized as follows [13]:

1. Resource:

a. Renewable resources;

b. Non-renewable resources;

c. Doubly constrained resources (e.g., money and energy).

2. Characteristics of activity:
a. Preemption;
b. Execution mode; c. Type of resource consumption during processing
of an activity;
d. Other characteristics.

3. Objective function:
a. Time-based;
b. Economic;
c. Resource-based;
d. Multi-objective;
e. Other objectives.

4. Availability level of information:
a. Deterministic;
b. Non-deterministic.

Due to NP-hardness of RCPSP and in order for its better adaptation to the reality, some researchers have considered various extensions and different methods for solving the problem. In part, they have worked on exact methods using mixed-integer programming, constraint programming, and satisfiability modulo theories [14-22]. Bibiks et al. [23] presented a Discrete Cuckoo Search (DCS) algorithm for RCPSP and improved the DCS algorithm in their study [24]. Fathallahi and Najafi [25] discussed a fuzzy RCPSP with the aim of maximizing Net Present Value (NPV) of project cash flows. They proposed a mathematical formulation and a hybrid Genetic Algorithm (GA) to solve the problem in hand. Gonzalez-Pardo et al. [26] modeled the RCPSP by constraint satisfaction problems-graph and solved it by ant colony optimization. Chand et al. [1] dealt with a genetic programming based hyper-heuristic to produce efficient priority rules for the RCPSP. Kadri and Boctor [27] addressed the RCPSP with transfer times to make them known and deterministic. To solve the problem, they proposed a GA. Coelho and Vanhoucke [28] developed a branchand-bound procedure to solve the RCPSP.

Multi-mode Resource-Constrained Project Scheduling Problem (MRCPSP) is an extensions of the RCPSP for which, because of its more complexity, different common methods have been presented in the literature [29-32]. Fernandes Muritiba et al. [33] developed a Path-Relinking (PR) algorithm for MRCPSP to minimize makespan of the project. Van Den Eeckhout et al. [34] integrated multi-mode RCPSP and resource scheduling in order to introduce some flexibilities into the scheduling process to determine the optimal personnel budget that minimized the overall cost.

It is possible to use manpower with different skills in the implementation of projects, which can be considered for the RCPSP as another generalization and it is called Multi-Skill Resource-Constrained Project Scheduling Problem (MSRCPSP) [35-38]. Javanmard et al. [39] integrated MSRCPSP with the resource investment problem to obtain concurrent optimal policy of project scheduling and recruitment of skills. Wang and Zheng [40] proposed a multi-objective fruit fly optimization for the MSRCPSP with the criteria of minimizing the makespan and total cost, simultaneously. Myszkowski et al. [41] designed a hybrid differential evolution and greedy algorithms for the MSRCPSP.

Since the choice of technology and the implementing route of different parts of a project can be an important factor that will lead to success or failure of the project in reality, in this paper, the RCPSP is generalized and project flexibility is considered for the choice of different technologies. Regarding different types of technology, different routes are considered for each section. Each route has independent activity set, precedence relationships, cost, and separated time for each activity. Therefore, in addition to sequencing of activities by limited operational resources, choosing the optimal route for the implementation of different parts of the project is important, which leads to a more complicated problem. In this paper, such problems are summarized as RCPSP-MR. The major difference between RCPSP-MR and MRCPSP is that in the MRCPSP, the project activity network (i.e., the number of activities and precedence relationships) is fixed, duration of each activity is varied with regard to the number of resource allocations, and all project activities are essential. On the other hand, in RCPSP-MR, the selection of a route may lead to basic changes in the project so that each part of the project activity network is replaced with a different activity sub-network. This leads to some changes in the project in terms of: 1) the number of activities, 2) precedence relationships between activities, 3) allocation of resources to the activities, 4) duration of each activity, and 5) cost of each activity, which increase the complexity of the problem.

RCPSP-MR problems can be encountered in the scheduling of projects, e.g., mechanizing vari- 
ous production lines, launching smart urban systems, dispatching liquid reservoir fuel, launching different vehicle tracking systems, developing educational simulators, and developing electronic Siebel for shooting training and reverse engineering of critical and important equipment. Also, RCPSP-MR is defined and described in this paper as indicated in flexible manufacturing cells according to the related literature [e.g., $42-55]$.

To solve the mathematical model in this paper, two meta-heuristic algorithms are proposed. First, in the proposed GA, due to the importance of producing initial solutions, each chromosome in the problem space is divided into seven parts, among which two are considered for the sequencing of the total project activities (e.g., fixed and flexible activities), one is related to route and selected for the flexible parts, and four are devoted to the assigned reassurances and the level of the implementation for activities. Solutions are reinforced with crossover and mutation operations frequently and, in each iteration, a specification of high quality solutions is saved. Second, considering that the solution space presented for the problem is discrete and the proposed Particle Swarm Optimization (PSO) algorithm is used in a continuous space, it is necessary to use an appropriate method to transform the production solutions into the continuous space. The method used in this paper is as follows: for each number of project activities, an equal interval between 0 to1 is considered. The selection of the numbers inside each interval in each iteration indicates the selection of the corresponding activity. Also, in the proposed algorithm, in order to produce quality solutions, mutation is considered in the presented GA algorithm and the initial solution is presented. According to the velocity vector in each iteration, the obtained solutions with the proposed algorithm move towards the optimal solution.

In Section 2, a mathematical model to solve the RCPSP-MR problem is presented. Since software packages for the proposed model are not able to find optimal solutions for medium- and large-size problems, in Section 3, two solution algorithms based on GA and PSO are presented to achieve near-optimal solutions. Then, in Section 4, 50 test problems are designed and solved with the proposed algorithms and the results are compared with each other. Conclusions are provided in Section 5 .

\section{RCPSP with multiple routes for flexible project activities}

\subsection{Problem description}

In this paper, a mixed-integer nonlinear programming model is proposed to find an optimal solution for the RCPSP-MR. The activity network is CPM and Activity On Node (AON) divided into two main sec- tions of flexible and fixed activities. The flexible activities are the part of the project to which, due to the ability of using various technologies in their implementation, different routes can be assigned. The route selection leads to the addition of new activity subnetworks to the main network of the project, making it broader and more complicated in decision-making on implementation.

A graph $G\left(J, A_{J}\right)$ is considered in witch $J=$ $\{0,1,2, \ldots, N+1\}$ is the list of activities ( 0 and $N+1$ are the artificial start and the end of the project, respectively) and $\left\{A_{J} \mid \forall(i \rightarrow j) \in A_{J}\right\}$ is the represented list of Finish-to-Start (FS) precedence relationships.

The duration of each activity is shown with $d$, which is not preemptive. Two sets of resources are considered for the problem, non-renewable $N R$, and multi-skill renewable $R$. The resources are available until the completion of the whole project and they are not transferable to other projects. Levels of the implementation for activity $j$ by the renewable resource $r \in R$ are shown by $l_{r} \in L_{r}$, where $L_{r}$ is the total number of execution levels. The purpose of the problem introduced in this paper is to minimize the cost of project completion, comprising: 1) total cost of the availability of renewable resources until the project completion, 2) total cost of using renewable resources and the level of implementation for any project activity, and 3) total cost of using non-renewable resources for project activities. All the sets, indices, and variables are presented as follows:

\section{Sets and indices:}

$J \quad$ Set of total project activities $J=\{0,1,2, \ldots, N+1\}$

$J_{1} \quad$ Set of total project fixed activities $J_{1} \subseteq J$

$J_{2} \quad$ Set of total flexible project activities $J_{2}=J-J_{1}$

$\lambda_{\rho} \quad$ Set of routes of each flexible activity $\rho \in J_{2}$

$\rho_{h} \quad$ Set of total sub-networks for flexible activities $\rho_{h}=\left\{0,1,2, \ldots, N_{\rho_{h}}+1\right\}$

$A_{J} \quad$ Set of precedence relationships for $J$ in graph $G=\left(J, A_{J}\right)$

$A_{J_{1}} \quad$ Set of precedence relationships for $J_{1}$ in graph $G=\left(J_{1}, A_{J_{1}}\right)$

$A_{\rho_{k}} \quad$ Set of precedence relationships for $\rho_{h}$ in graph $G_{\rho_{h}}=\left(\rho_{h}, A_{\rho_{h}}\right)$

$R \quad$ Set of renewable resources

$N R \quad$ Set of non-renewable resources

$L_{r} \quad$ Set of levels of implementation for renewable resource $r$

$i, j \quad$ Index of activity 
$\rho, \rho, \quad$ Index of flexible activities

$h, h^{\prime} \quad$ Index of route

$l \quad$ Index of level of implementation

\section{Parameters:}

$\gamma_{j n r} \quad$ Non-renewable resource requirement for fixed activity $j$

$\gamma_{j \rho_{h} n r} \quad$ Non-renewable resource requirement for activity $j$ of flexible part $\rho_{h}$

$C \quad$ Fixed cost of the availability of renewable resources per unit of time

$C_{j r l} \quad$ Cost of using renewable resources and its level of implementation for activity j

$C_{j r l \rho_{h}} \quad$ Cost of using renewable resources and its level of implementation for activity $j$ of flexible part $\rho_{h}$

$C_{n r} \quad$ Fixed cost of using non-renewable resources

$d_{j r l} \quad$ Duration of using renewable resources and the level of implementation for activity $j$

$d_{j r l \rho_{h}} \quad$ Duration of using renewable resources and the level of implementation for activity $j$ of flexible part $\rho_{h}$

\section{Binary variables:}

$Y_{j r l}=\{0,1\} \quad$ Level of implementation for activity $j$ by renewable resource $r$

$Y_{j r l \rho_{h}}=\{0,1\}$ Level of implementation for activity $j$ by renewable resource $r$ of flexible part $\rho_{h}$

$W_{\rho_{h}}=\{0,1\} \quad$ Route selection $h$ for flexible activity $\rho$ $\alpha_{r j i}=\{0,1\} \quad$ Sequencing of fixed activities $i$ and $j$ allocated to resource $r$ considering the precedence relationships

$\eta_{r j i \rho_{h}}=\{0,1\}$ Sequencing of flexible activities $i$ and $j$ of flexible part $\rho_{h}$ allocated to resource $r$ considering the precedence relationships

$\psi_{r j \rho_{h} i \rho^{\prime} h^{\prime}}=\{0,1\}$ Sequencing of flexible activity $j$ of flexible part $\rho_{h}$ and flexible activity $i$ of flexible part $\rho_{h^{\prime}}^{\prime}$ allocated to resource $r$ considering the precedence relationships

\section{Positive variables:}

$F_{j r l} \quad$ Finish time activity $j$ allocated to resource $r$ at level $l$

$S_{j r l} \quad$ Start time activity $j$ allocated to resource $r$ at level $l$

$F_{j r l \rho_{h}} \quad$ Finish time activity $j$ of flexible part $\rho_{h}$ allocated to resource $r$ at level $l$
$S_{j r l \rho_{h}} \quad$ Start time activity $j$ of flexible part $\rho_{h}$ allocated to resource $r$ at level $l$

TC Total Cost of project

\subsection{Mathematical formulation}

The proposed mathematical formulation for the problem is given as follows:

$$
\begin{aligned}
& \operatorname{Min} T C=C \cdot \sum_{r \in R} \sum_{l \in L_{r}}\left(F_{(N+1) r l} \cdot Y_{(N+1) r l}\right) \\
& +\sum_{j \in J_{1}} \sum_{r \in R} \sum_{l \in L_{r}}\left(C_{j r l} . Y_{j r l} \cdot d_{j r l}\right) \\
& \left.+\sum_{\rho \in J_{2}} \sum_{h \in \lambda_{\rho}} \sum_{j \in \rho_{h}} \sum_{r \in R l \in L_{r}} \sum_{j r l \rho_{h}} \cdot Y_{j r l \rho_{h}} \cdot d_{j r l \rho_{h}} \cdot W_{\rho_{h}}\right) \\
& +\sum_{n r \in N R} C_{n r} \cdot\left(\sum_{\rho \in J_{2}} \sum_{h \in \lambda_{\rho}} \sum_{j \in \rho_{h}}\left(\gamma_{j \rho_{h} n r} \cdot W_{\rho_{h}}\right)\right. \\
& \left.+\sum_{j \in J_{1}} \gamma_{j n r}\right),
\end{aligned}
$$

Subject to:

$$
\begin{aligned}
& \sum_{r \in R} \sum_{l \in L_{r}}\left(F_{j r l} . Y_{j r l}\right) \geq\left(\sum_{r \in R} \sum_{l \in L_{r}}\left(F_{i r l} . Y_{i r l}\right)\right. \\
& \left.\quad+\sum_{r \in R} \sum_{l \in L_{r}}\left(d_{j r l} . Y_{j r l}\right)\right), \quad \forall(i, j) \in A_{J_{1}},
\end{aligned}
$$

$\sum_{r \in R} \sum_{l \in L_{r}}\left(F_{j r l \rho_{h}} \cdot Y_{j r l \rho_{h}}\right) \cdot W_{\rho_{h}}$

$$
\begin{aligned}
& \geq\left(\sum_{r \in R} \sum_{l \in L_{r}}\left(F_{i r l \rho_{h}} . Y_{i r l \rho_{h}}\right)\right. \\
& \left.+\sum_{r \in R} \sum_{l \in L}\left(d_{j r l \rho_{h}} . Y_{j r l \rho_{h}}\right)\right) \cdot W_{\rho_{h}},
\end{aligned}
$$$$
\forall \rho \in J_{2}, \forall h \in \lambda_{\rho}, \forall(i, j) \in A_{\rho_{h}},
$$

$\sum_{r \in R} \sum_{l \in L_{r}}\left(F_{j r l} . Y_{j r l}\right) \geq\left(\sum_{r \in R} \sum_{l \in L_{r}}\left(F_{i r l \rho_{h}} . Y_{i r l \rho_{h}} . W_{\rho_{h}}\right)\right.$

$$
\left.+\sum_{r \in R} \sum_{l \in L_{r}}\left(d_{j r l} . Y_{j r l}\right)\right)
$$$$
\forall \rho \in J_{2}, \forall(\rho, j) \in A_{J}, \forall h \in \lambda_{\rho}, \forall i \in \rho_{h},
$$

$\sum_{r \in R} \sum_{l \in L_{r}}\left(Y_{j r l} \cdot\left(F_{j r l}-S_{j r l}-d_{j r l}\right)\right)=0, \quad \forall j \in J_{1}$, 


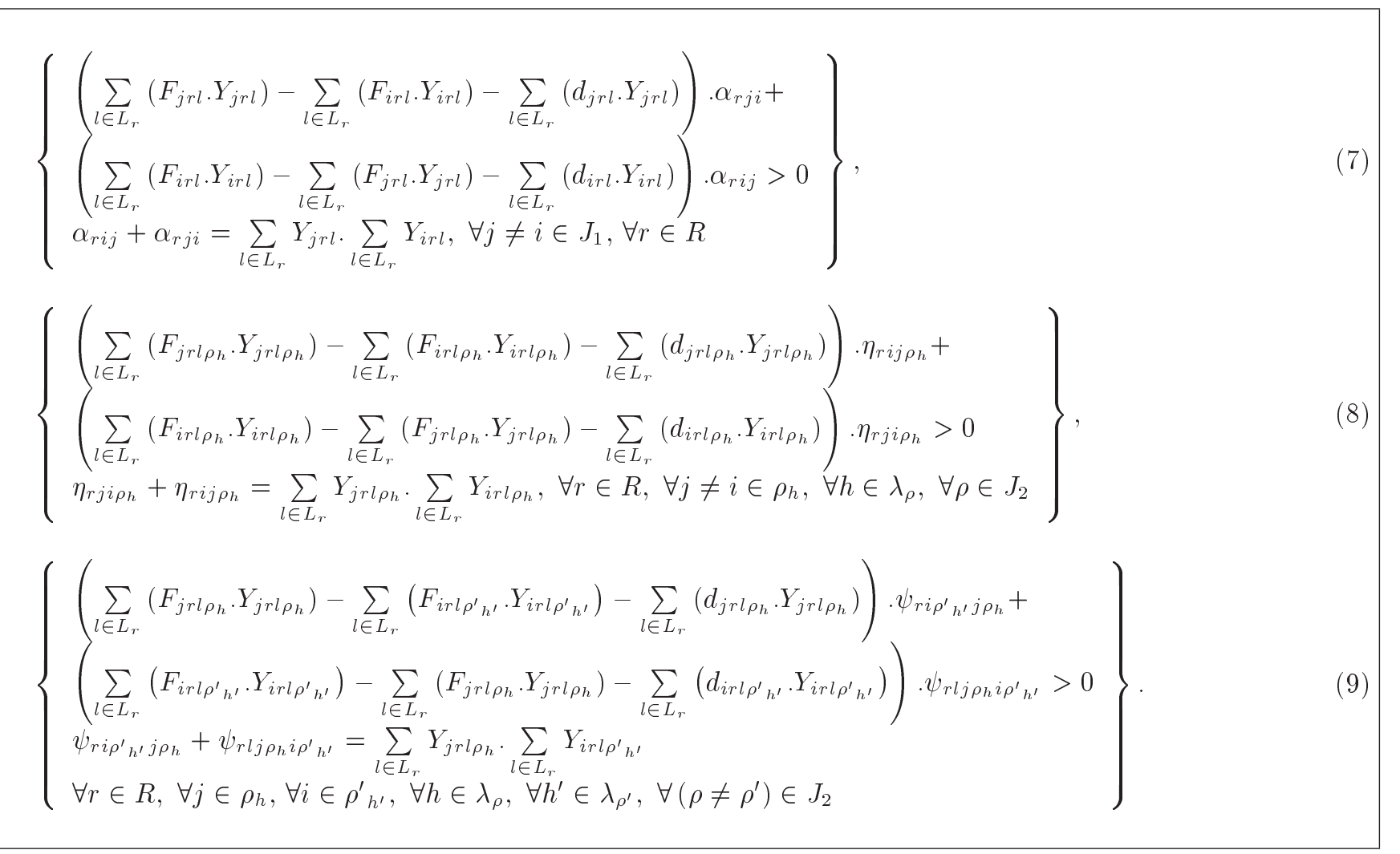

Box I

$$
\begin{gathered}
W_{\rho_{h}} \cdot \sum_{r \in R} \sum_{l \in L_{r}}\left(Y_{j r l \rho_{h}} \cdot\left(F_{j r l \rho_{h}}-S_{j r l \rho_{h}}-d_{j r l \rho_{h}}\right)\right)=0, \\
\quad \forall \rho \in J_{2}, \forall h \in \lambda_{\rho}, \forall j \in \rho_{h} .
\end{gathered}
$$

Eqs. (7)-(9) are shown in Box I.

$$
\begin{aligned}
& \sum_{r \in R} \sum_{l \in L_{r}} Y_{j r l}=1, \forall j \in J_{1}, \\
& \sum_{r \in R} \sum_{l \in L_{r}} Y_{j r l \rho_{h}}=W_{\rho_{h}}, \forall \rho \in J_{2}, \forall j \in \rho_{h}, \forall h \in \lambda_{\rho}, \\
& \sum_{h \in \lambda_{\rho}} W_{\rho_{h}}=1, \forall \rho \in J_{2} .
\end{aligned}
$$

Eq. (1) is the objective function by which the total cost of project completion is minimized. Relations (2)(4) assure that the completion time of each activity is greater than (or equal to) the completion time of its predecessor activities. Eqs. (5) and (6) indicate that each activity must be implemented without interruption. Relations (7)-(9) define relations between the completion times of each pair of activities by each renewable resource. Eqs. (10) and (11) explain that a project activity will be carried out only by an executive level of renewable resources. Eq. (12) guarantees that only one route out of the possible routes is selected for each flexible activity $\rho \in J_{2}$.

\section{Proposed meta-heuristic algorithms}

\subsection{Genetic Algorithm (GA)}

GA is a competitive algorithm among other metaheuristic algorithms in which the presence or absence of genes and their order in the chromosome decide the characteristics of a species. Different traits are passed from generation to the next through different biological processes that operate on the genetic structure [56,57]. By the process of genetic change, the fittest population may well adapt to the environment $[58,59]$. GA has attracted the attention of researchers for use in the PSP and its extensions $[60,61]$.

The first proposed meta-heuristic algorithm for the RCPSP-MR problem in this study is presented based on the GA, which is abbreviated as GA-MR hereinafter. In this algorithm, with regard to the dimensions of the problem, a certain number of initial solutions are generated.

Generation of the initial solution is in a way that each solution (chromosome) consists of different parts:

1. C.Main (C.Flex), which determines the permutation of all fixed activities of the main project network (or all activities related to any flexible activity) by observing all the precedence relations;

2. C.R (C.FR), which determines the resource allo- 
cated to each fixed activity (or each sub-activity that is related to the flexible activity network);

3. C.L (C.FL), which determines the level of implementation for any fixed activity (or any sub-activity that is related to the flexible activity network) by the allocated resources to the previous part;

4. C.Route, which determines the route selected for each flexible activity.

By calculating the total cost of project completion based on the roulette wheel, the fitness value or generation solution value will be calculated by Eq. (13).

$$
P_{i}=1-\frac{T C_{i}}{\sum_{i=1}^{N} T C_{j}},
$$

where $T C_{i}$ is the objective function (total project cost) in solution $i$. As shown in Eq. (13), the goodness ratio of one solution or chromosome $\left(P_{i}\right)$ is calculated based on the goodness value of all the obtained solutions. Higher ratios will raise the chance of chromosome selection for the next solution production. The most valuable solutions will be selected. Then, crossover operation is implemented. The crossover location is randomly chosen and the number of new solutions from each parent is in accordance with Eq. (14):

$$
C H=\left(2+J_{2}\right) \times 2 .
$$

In Eq. (14), $C H$ indicates the number of new solutions and $J_{2}$ indicates the number of flexible activities. For example, consider a network with the number of eight activities having a flexible activity. For the flexible activity, two separate routes are considered. The main project activity network and the sub-networks of flexible activity route are shown in Figure 1.

The crossover operation is performed on $C . R$, C.L, C.Route, C.FR, and C.FL parts of the solution considered in Figure 2.
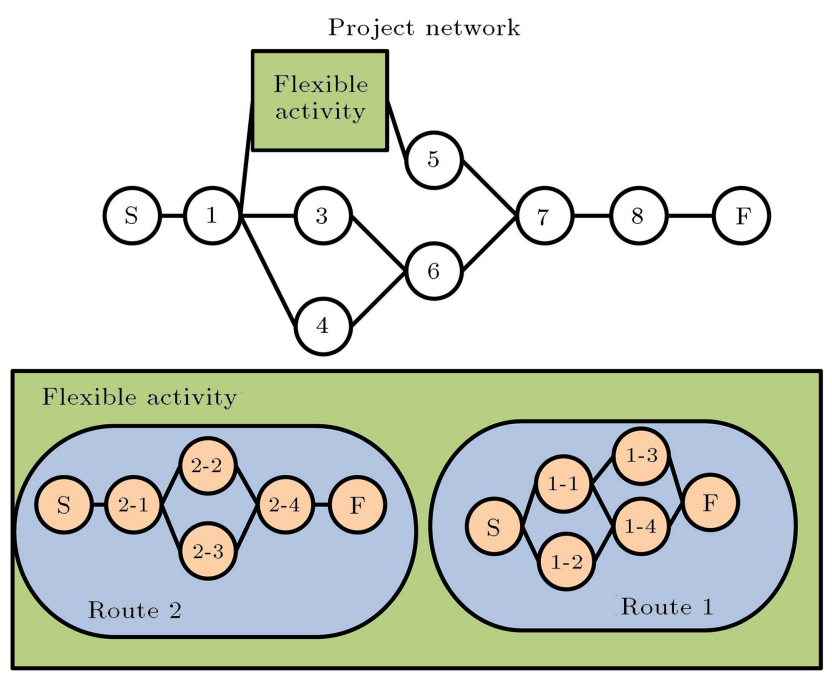

Figure 1. Project activity network.
In the next step, using the four different types of mutation for the most valuable solutions of the crossover operation, the new solutions are generated and their values are calculated. The number of new solutions from mutation operations follows Eq. (15):

$$
C H=1+4 J_{2} \text {. }
$$

The various types of mutation are: 1) swap mutation, 2) flip mutation, 3) reversion mutation, and 4) insertion mutation, which are performed on C.R, C.L, C.Route, C.FR, and C.FL parts of the solution considered in Figure 3 .

1. Swap mutation: In this mutation, two positions of the chromosomes are randomly selected and their corresponding values are exchanged. In this paper, the mutation is done on C.Main and C.Flex;

2. Reversion mutation: In this mutation, two points are randomly selected from the parent chromosome and the numbers are written between these two points inversely from the end to the beginning. In this paper, the mutation is carried out on C.FR and C.FL;

3. Insertion mutation: In the insertion mutation, two points of the chromosome are selected and their positions are changed. After random selection of two points of the parent chromosome, the second point is transferred next to the first point. In this paper, the mutation is done on C.FR and C.FL;

4. Flip mutation: In this mutation, a small impact is made on the chromosome. As a result, all the numbers will move forward up to the impact. Obviously, the numbers that are located at the end of the chromosome should be transferred to its beginning with regard to the flip rate. In this paper, the mutation is done on C.FR and C.FL.

Finally, all the generation solutions are categorized and the certain number of best solutions are stored in a memory. The above-mentioned process is repeated to a certain number and after acquisition of the stop conditions, the best route selected for each flexible activity, the best permutation determined for network activities, the best resource allocation conditions, and the level of implementing each activity will be provided in the form of the best solution. In Figure 4, steps of the GA-MR algorithm are briefly presented in a flowchart.

\subsection{Particle Swarm Optimization (PSO)}

Particle Swarm Optimization (PSO) was developed by a social psychologist named Kennedy and an electrical engineer named Eberhart [62]. The PSO algorithm consists of initial solutions or particles with separate position and velocity vectors [63]. The particles move 


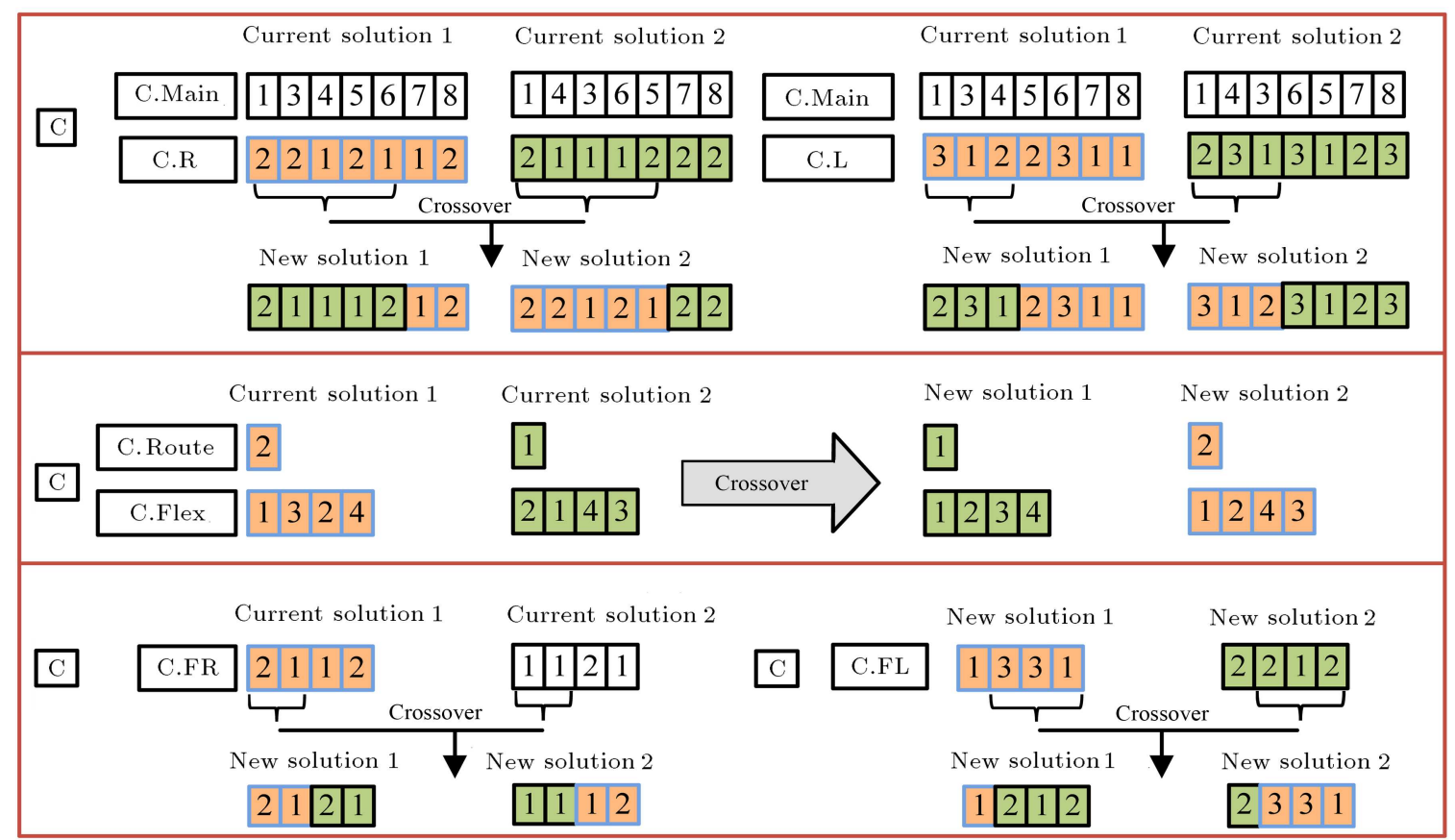

Figure 2. Crossover operation on a solution.

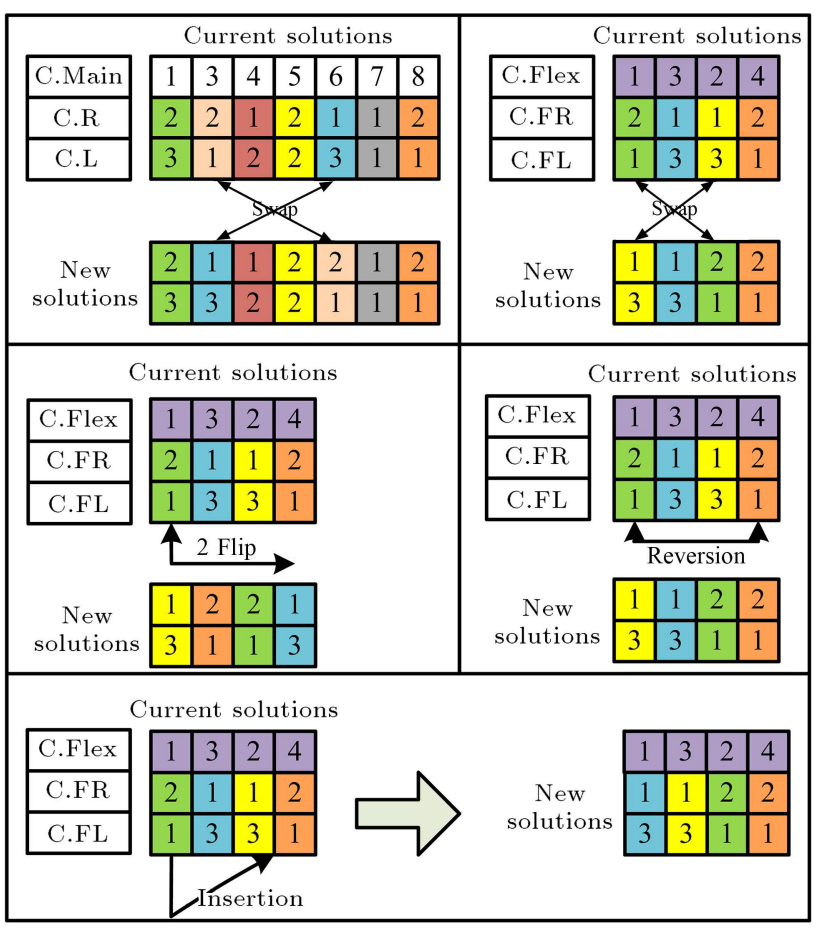

Figure 3. Types of mutation operations.

frequently in the problem space with regard to the velocity vector in each iteration and examine the new possible options by calculating the fitness value as a standard measurement. Two memories are assigned to storing the best position of each particle in the past (the best local solution) and the best position among all particles (the best global solution). Finally, by considering the stopping algorithm conditions, the best global solution is determined as the result of the algorithm [64,65].

The velocity and position vectors of each particle are updated as follows [66]:

$$
\begin{aligned}
& \vec{v}_{k}^{i}=\delta \cdot\left(w \cdot \vec{v}_{k-1}^{i}+c_{1} \cdot \vec{r}_{1} \cdot\left(\vec{p}_{k}^{i}-\vec{x}_{k}^{i}\right)+c_{2} \cdot \vec{r}_{2} \cdot\left(\vec{p}_{k}^{q}-\vec{x}_{k}^{i}\right)\right), \\
& \vec{x}_{k+1}^{i}=\left\lfloor\vec{x}_{k}^{i}+\vec{v}_{k}^{i}\right\rfloor,
\end{aligned}
$$

where for particle $i, \vec{x}_{k}^{i}$ is position vector, $\vec{v}_{k}^{i}$ is velocity vector, $\vec{p}_{k}^{i}$ is the best position vector, and $\vec{p}_{k}^{q}$ is the best of position vector between all particles in iteration $k$. The vectors $\overrightarrow{r_{1}}$ and $\overrightarrow{r_{2}}$ are random numbers distributed between 0 and 1 . $c_{1}$ and $c_{2}$ are acceleration constants for the local and global searches [67]. The parameter is the inertial weight factor and its values are different at each step of algorithm iteration calculated by Eq. (18):

$$
w=W_{\max }-\left(\frac{W_{\max }-W_{\min }}{N}\right) \times k,
$$

where $k$ is the current iteration index and $N$ is the total number of iterations in the algorithm. $W_{\min }$ and $W_{\max }$ are constants [66]. The parameter $\delta$ is contraction coefficient for velocity control obtained through Eq. (19):

$$
\delta=\left(\frac{2}{\left|C-2+\sqrt{C^{2}-4 C}\right|}\right), C=c_{1}+c_{2}, \quad C \geq 4 .
$$

The flowchart of the proposed improved algorithm is depicted in Figure 5 based on the PSO, called PSOMR. 


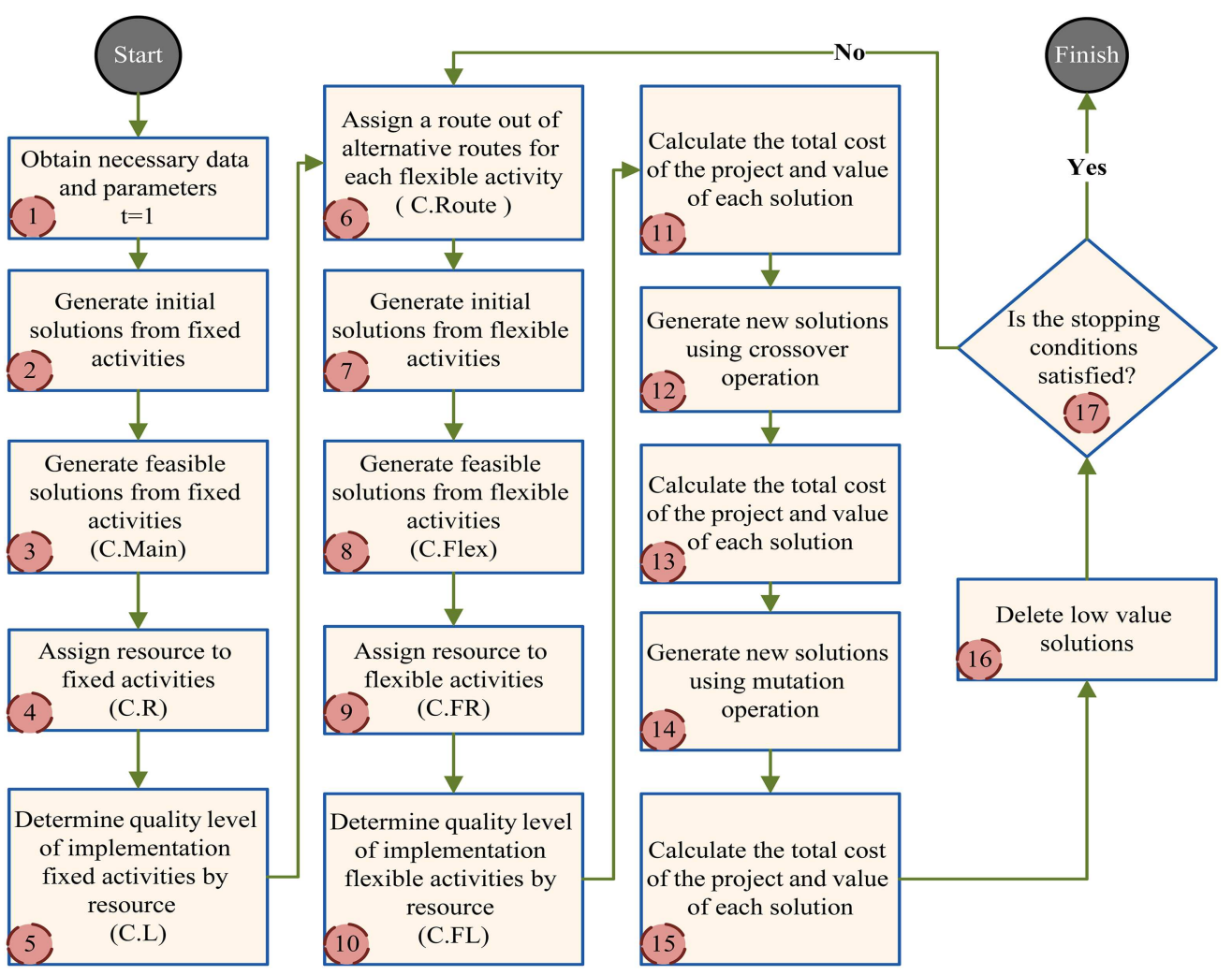

Figure 4. Flowchart of the proposed GA-MR.

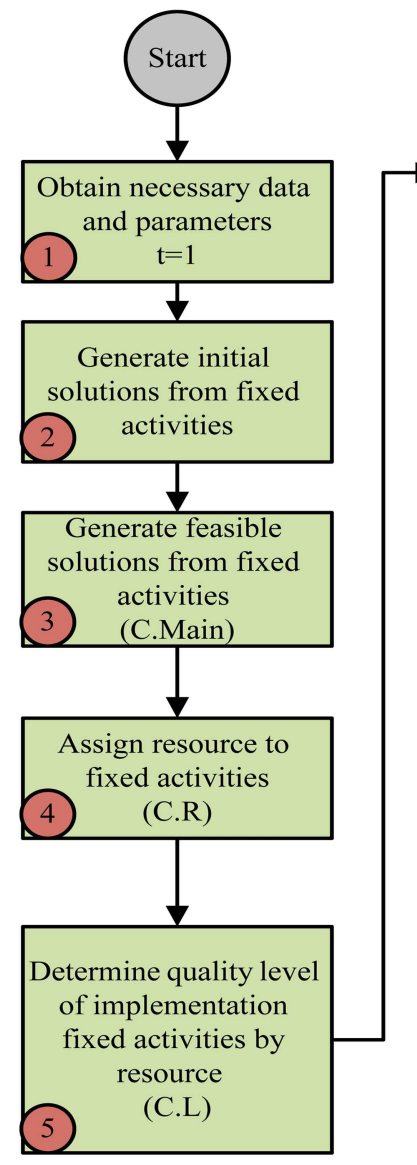

Assign a route out of alternative routes for each flexible activity (6) ( C.Route)

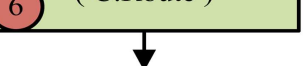

Generate initial solutions from flexible activities

(7)

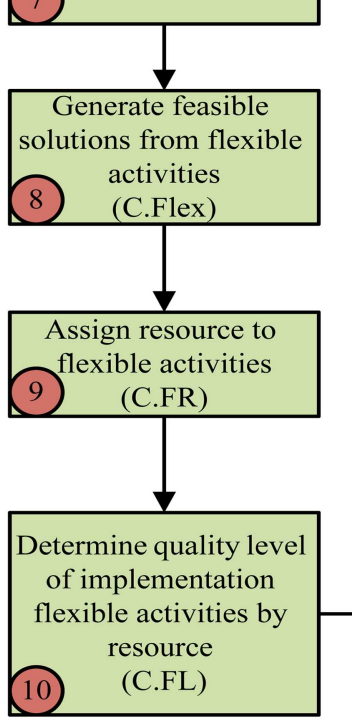

Figure 5. Flowchart of proposed PSO-MR. using mutation

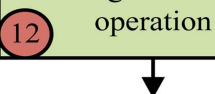
13 Generate initial
velocity vectors

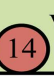

(11)

Calculate the objectiv

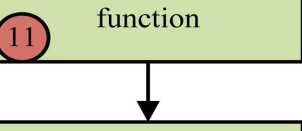

Generate new solutions

Calculate the objective
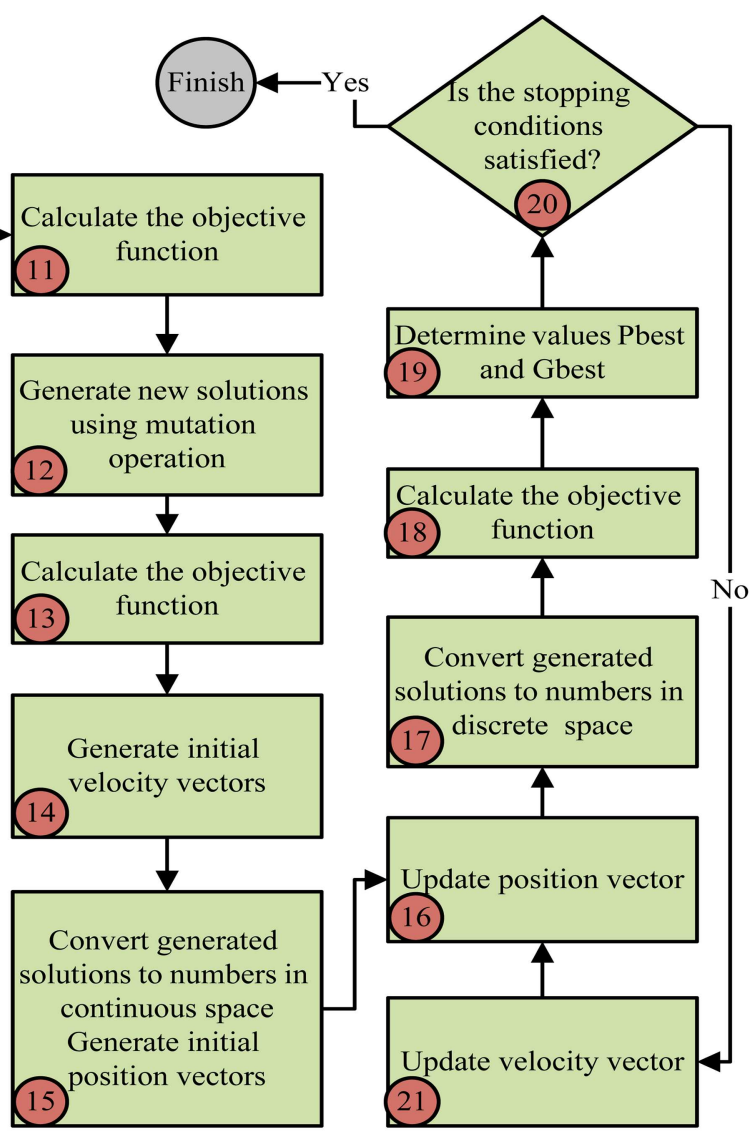

(21) 


\subsection{Numerical example}

To evaluate the performance of the presented mathematical model, a small-scale example is designed and solved with the proposed model. In this example, consider an AON network of a project with three activities, $J=\{0,1,2,3,4\}$, where activity 1 is flexible, $J_{2}=\{1\}$, and other activities are fixed, $J_{1}=\{0,2,3,4\}$. For flexible activity 1 , three different routes are considered, $\lambda_{2}=\{1,2,3\}$. The network of activities of each route is presented in Figure 6 . For the implementation of all project activities, only a renewable resource, $R=\{1\}$, is considered with two levels of implementation, $L_{1}=\{1,2\}$, and two types of non-renewable resources, $N R=\{1,2\}$. The values
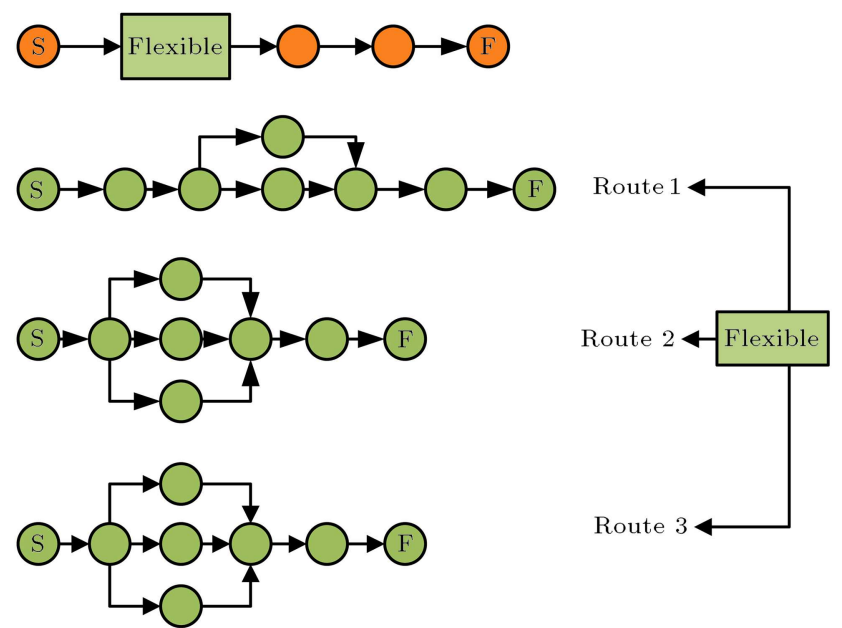

Route 3

Figure 6. Network of activities in the numerical example. for any activity are individually specified in Table 1. The cost of using any unit of non-renewable resources is $C_{1}=10$, and $C_{2}=8$.

Other information on the problem can be seen in Table 1.

The problem was formulated using Eqs. (1)-(12) and solved by the GAMS optimization software. The numbers of equations and variables used to solve the problem were 973 and 528, respectively. Route 2 for flexible activity 1 was selected and optimal solution 1654 was obtained in 248.65 s. In Figure 7 , sequencing of activities is shown.

The numerical example was then solved with the proposed GA-MR and PSO-MR. The results obtained are shown in Figure 7. The optimal solution 1654 was obtained by GA-MR in $7.23 \mathrm{~s}$ and by PSO-MR in $11.51 \mathrm{~s}$, respectively.

\section{Computational results}

In this paper, in order to test the performance of the proposed mathematical model and algorithms, a number of 50 problems in various scales (20 very small problems, 10 small problems, 10 medium problems, and 10 large sized problems) are designed and solved. As shown in Table 2, all the designed problems are marked with a certain ID from 001 to 050 . The first 20 problems (from 001 to 020 ) are very small, 10 (from 021 to 030 ) are small, 10 (from 031 to 040) are medium, and 10 (from 041 to 050) are large sized problems. Also, each problem is identified through a

Table 1. Total information of the first numerical example.

\begin{tabular}{|c|c|c|c|c|c|c|c|c|c|c|c|c|c|c|c|c|}
\hline \multicolumn{17}{|c|}{ Route selection 1 for flexible activity 1} \\
\hline Activity & \multicolumn{2}{|c|}{1} & \multicolumn{2}{|c|}{2} & \multicolumn{2}{|c|}{3} & \multicolumn{2}{|c|}{4} & \multicolumn{2}{|c|}{5} & \multicolumn{2}{|c|}{6} & \multicolumn{2}{|c|}{7} & \multicolumn{2}{|c|}{8} \\
\hline$r$ & \multicolumn{2}{|c|}{1} & \multicolumn{2}{|c|}{1} & \multicolumn{2}{|c|}{1} & \multicolumn{2}{|c|}{1} & \multicolumn{2}{|c|}{1} & \multicolumn{2}{|c|}{1} & \multicolumn{2}{|c|}{1} & \multicolumn{2}{|c|}{1} \\
\hline$l$ & 1 & 2 & 1 & 2 & 1 & 2 & 1 & 2 & 1 & 2 & 1 & 2 & 1 & 2 & 1 & 2 \\
\hline$d$ & 8 & 12 & 10 & 11 & 14 & 13 & 13 & 15 & 11 & 13 & 15 & 10 & 13 & 15 & 14 & 12 \\
\hline$C$ & 7 & 7 & 7 & 7 & 6 & 7 & 10 & 7 & 6 & 6 & 7 & 7 & 7 & 6 & 9 & 7 \\
\hline$n r$ & 1 & 2 & 1 & 2 & 1 & 2 & 1 & 2 & 1 & 2 & 1 & 2 & 1 & 2 & 1 & 2 \\
\hline$\gamma$ & 0 & 0 & 1 & 2 & 4 & 2 & 3 & 5 & 5 & 0 & 4 & 3 & 1 & 5 & 2 & 1 \\
\hline \multicolumn{17}{|c|}{ Route selection 2 for flexible activity 1} \\
\hline$d$ & 11 & 8 & 9 & 12 & 13 & 10 & 10 & 10 & 14 & 9 & 8 & 10 & 13 & 15 & 14 & 12 \\
\hline$C$ & 10 & 7 & 6 & 9 & 8 & 9 & 9 & 9 & 9 & 9 & 6 & 8 & 7 & 6 & 9 & 7 \\
\hline$n r$ & 1 & 2 & 1 & 2 & 1 & 2 & 1 & 2 & 1 & 2 & 1 & 2 & 1 & 2 & 1 & 2 \\
\hline$\gamma$ & 1 & 1 & 0 & 0 & 1 & 3 & 2 & 5 & 1 & 3 & 3 & 2 & 1 & 5 & 2 & 1 \\
\hline \multicolumn{17}{|c|}{ Route selection 3 for flexible activity 1} \\
\hline$d$ & 15 & 8 & 15 & 8 & 12 & 12 & 12 & 10 & 14 & 8 & 12 & 8 & 13 & 15 & 14 & 12 \\
\hline$C$ & 9 & 7 & 6 & 8 & 8 & 7 & 7 & 7 & 6 & 7 & 6 & 10 & 7 & 6 & 9 & 7 \\
\hline$n r$ & 1 & 2 & 1 & 2 & 1 & 2 & 1 & 2 & 1 & 2 & 1 & 2 & 1 & 2 & 1 & 2 \\
\hline$\gamma$ & 3 & 3 & 2 & 5 & 0 & 3 & 3 & 2 & 0 & 3 & 2 & 5 & 1 & 5 & 2 & 1 \\
\hline
\end{tabular}




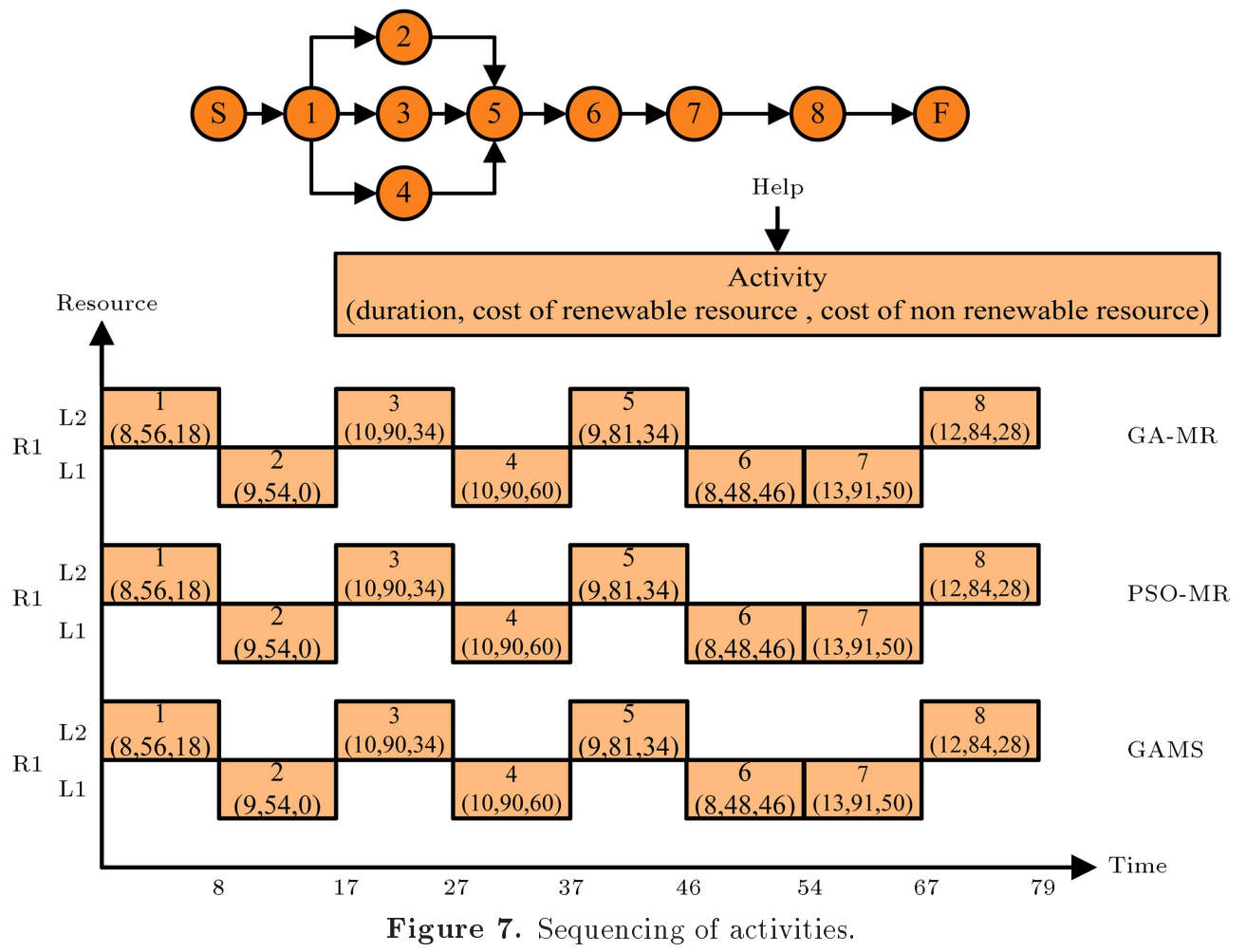

Table 2. General characteristics of the designed problems.

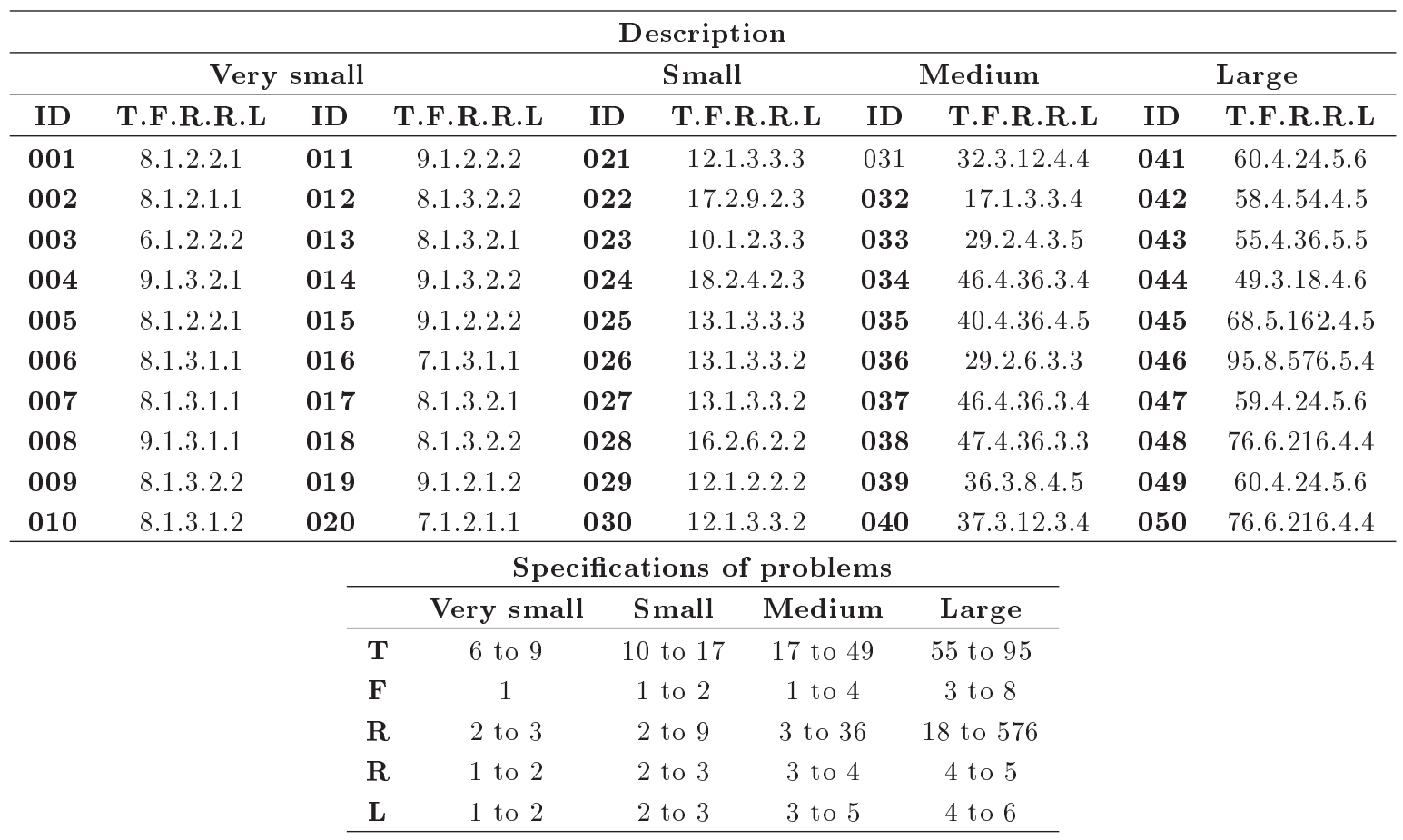

specific T.F.R.R.L label. In this ID, $T$ is the lowest total number of activities when adding new activity networks from a selection route for flexible project activities, $F$ is the number of flexible activities considered for the project, firstly, $R$ represents the number of applicable activity networks to the project (number of routes), secondly, $R$ represents the number of multiskill renewable resources considered for the project, and finally $L$ represents the number of application levels to perform any activities by renewable resources. In Table 2, characteristics of the designed problems are presented. 
Table 3. Results obtained by problem solving through the mathematical model as well as Particle Swarm Optimization with Multiple Routes (PSO-MR) and Genetic Algorithm with Multiple Route (GA-MR).

\begin{tabular}{cccccccccc}
\hline \multicolumn{2}{c}{ Description } & \multicolumn{2}{c}{ GAMS } & \multicolumn{2}{c}{ PSO-MR } & \multicolumn{2}{c}{ GA-MR } & \multicolumn{2}{c}{ Gap\% } \\
\hline ID & T.F.R.R.L & Time (s) & TC & Time (s) & TC & Time (s) & TC & PSO-MR & GA-MR \\
\hline $\mathbf{0 0 2}$ & 8.1 .2 .1 .1 & 20.74 & 1702 & 11.86 & 1702 & 7.87 & 1702 & 0 & 0 \\
$\mathbf{0 0 3}$ & 6.1 .2 .2 .2 & 533.3 & 1084 & 18.21 & 1084 & 17.35 & 1084 & 0 & 0 \\
$\mathbf{0 0 4}$ & 9.1 .3 .2 .1 & 2409 & 1552 & 12.52 & 1552 & 15.63 & 1552 & 0 & 0 \\
$\mathbf{0 0 6}$ & 8.1 .3 .1 .1 & 76.17 & 1411 & 11.48 & 1411 & 7.6 & 1411 & 0 & 0 \\
$\mathbf{0 0 7}$ & 8.1 .3 .1 .1 & 63.58 & 1769 & 11.47 & 1769 & 7.46 & 1769 & 0 & 0 \\
$\mathbf{0 0 9}$ & 8.1 .3 .2 .2 & 1444 & 1159 & 49.08 & 1159 & 29.28 & 1159 & 0 & 0 \\
$\mathbf{0 1 0}$ & 8.1 .3 .1 .2 & 248.65 & 1654 & 11.51 & 1654 & 7.23 & 1654 & 0 & 0 \\
$\mathbf{0 1 2}$ & 8.1 .3 .2 .2 & 991.71 & 1082 & 32.62 & 1098 & 17.92 & 1082 & 1.48 & 0 \\
$\mathbf{0 1 3}$ & 8.1 .3 .2 .1 & 717.4 & 1290 & 11.71 & 1290 & 13.97 & 1290 & 0 & 0 \\
$\mathbf{0 1 4}$ & 9.1 .3 .2 .2 & 1514 & 1404 & 87.09 & 1404 & 32.94 & 1404 & 0 & 0 \\
& Average & $\mathbf{8 0 1 . 8 5}$ & $\mathbf{1 4 1 0 . 7}$ & $\mathbf{2 5 . 7 5 5}$ & $\mathbf{1 4 1 2 . 3}$ & $\mathbf{1 5 . 7 2 5}$ & $\mathbf{1 4 1 0 . 7}$ & $\mathbf{0 . 1 4 8}$ & $\mathbf{0 . 0 0}$ \\
\hline
\end{tabular}

As seen in Table 2, the framework for very small, small, medium, and large sized problems is in such a way that $T$ for very small problems is in the range of 6-9, for small problems in the range of 10-17, for medium problems in the range of $17-49$, and for large problems in the range of 55-95. For example, suppose that the problem ID is 8.1.2.1.1. In this case, there will be a project with at least eight activities in the final network. In this project, there is also a flexible activity for the performance of which two different routes can be considered and the selection of each route leads to a new extra activity sub-network for the main network of the project. Therefore, the number of applicable activity networks for the project is two. The number of available multi-skill resources for implementing the project is equal to one and the number of quality levels applied to each activity by renewable resources is also considered equal to one.

\subsection{Comparing optimization software with the proposed GA-MR and PSO-MR}

In order to evaluate the performance of the mathematical model as well as GA-MR and PSO-MR metaheuristics, 10 small problems are considered and the results obtained by solving them are compared in terms of time and quality of the obtained solutions. As shown in Table 3, the framework of the designed problems is in such a way that $T$ is in the range of 6-9. Also, $F$ is equal to 1 , the first $R$ is in the range of $2-3$ and the second $R$ is in the range of $1-2$, and finally $L$ is in the range of $1-2$.

In Table 3, three columns are considered for the mathematical model and the proposed PSO-MR and GA-MR algorithms. In one of the columns, the data related to the best final solution are registered (TC columns) and another one represents the time required for reaching the best solution (Time (s) columns). TC is the estimated total cost for the implementation of the project, which is obtained from calculating the sum of three different costs. The project costs include: 1) the fixed cost of availability of resources during the project regardless of their uses for project activities; 2) the cost of using available renewable resources to carry out project activities with regard to the selection of proper levels for their applications, and 3) the cost of using non-renewable resources for project activities. Two other columns known as the gap (PSO-MR and GA-MR) are also considered. The gap represents the difference percentage of the PSO-MR and GA-MR solutions from the optimal solution. For example, if the gaps of the PSO-MR and GA-MR algorithms for a problem are $0.17 \%$ and $0.0 \%$, respectively, then the solution obtained by GA-MR is equal to the optimal solution, while PSO-MR obtains a solution $0.17 \%$ worse than the optimal solution.

As seen in Table 3 and Figure 8, the average solution obtained by modeling and solving in GAMS software is equal to 1410.7 in the average time of 801.85 s. The result obtained from the mathematical model is equal to that from GA-MR and better than that from PSO-MR with a difference of $0.148 \%$. Therefore, it can be stated that the presented mathematical model and the two meta-heuristics algorithms have good performance in terms of solution for very small problems. As seen in Table 3, the PSO-MR in the average time of $25.755 \mathrm{~s}$ obtains near-optimal solutions and GA-MR in the average time of $15.725 \mathrm{~s}$ obtains optimal solutions. Since the PSO-MR and GA- 


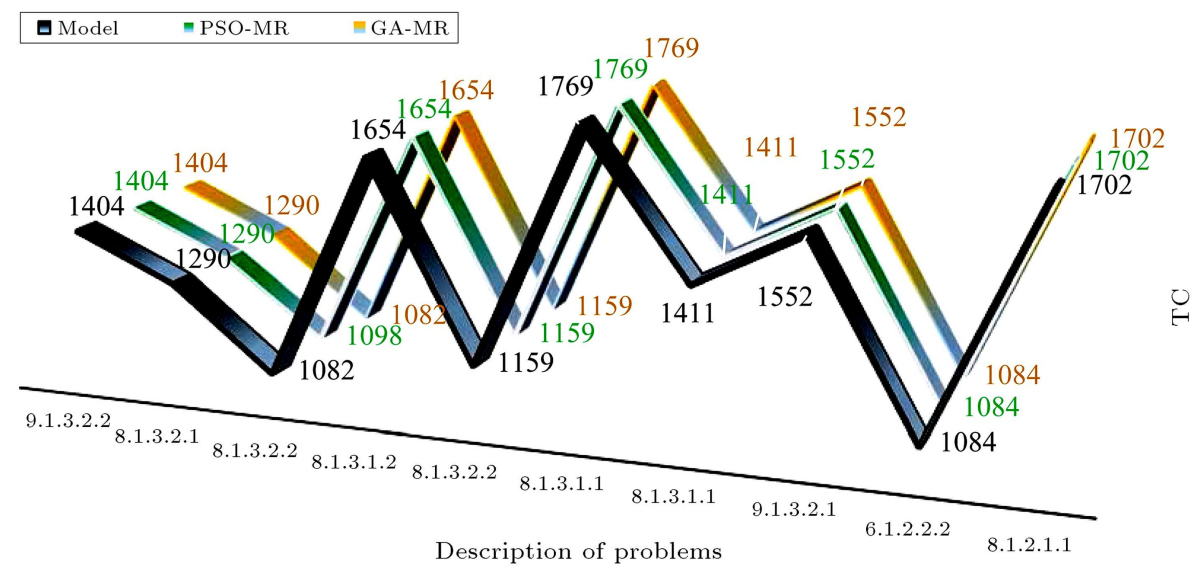

Figure 8. Results obtained through solving the problem by the mathematical model as well as Particle Swarm Optimization with Multiple Routes (PSO-MR) and Genetic Algorithm with Multiple Route (GA-MR).

Table 4. Computational results (very small problems).

\begin{tabular}{lcccccc}
\hline \multicolumn{7}{c}{ Very small sized problems } \\
\hline & \multicolumn{7}{c}{ PSO-MR } & \multicolumn{2}{c}{ GA-MR } & \multicolumn{2}{c}{ Best solution } \\
\cline { 2 - 7 } ID & Time (s) & TC & Time (s) & TC & Time & TC \\
\hline $\mathbf{0 0 1}$ & 22.62 & 1670 & 35.85 & 1670 & GA-MR & GA-MR \& PSO-MR \\
$\mathbf{0 0 2}$ & 11.86 & 1702 & 7.87 & 1702 & GA-MR & GA-MR \& PSO-MR \\
$\mathbf{0 0 3}$ & 18.21 & 1084 & 17.35 & 1084 & GA-MR & GA-MR \& PSO-MR \\
$\mathbf{0 0 4}$ & 12.52 & 1552 & 15.63 & 1552 & PSO-MR & GA-MR \& PSO-MR \\
$\mathbf{0 0 5}$ & 12.08 & 1629 & 8.05 & 1629 & GA-MR & GA-MR \& PSO-MR \\
$\mathbf{0 0 6}$ & 11.48 & 1411 & 7.60 & 1411 & GA-MR & GA-MR \& PSO-MR \\
$\mathbf{0 0 7}$ & 11.47 & 1769 & 7.46 & 1769 & GA-MR & GA-MR \& PSO-MR \\
$\mathbf{0 0 8}$ & 11.80 & 1576 & 8.29 & 1576 & GA-MR & GA-MR \& PSO-MR \\
$\mathbf{0 0 9}$ & 49.08 & 1159 & 29.28 & 1159 & GA-MR & GA-MR \& PSO-MR \\
$\mathbf{0 1 0}$ & 18.51 & 1654 & 7.23 & 1654 & GA-MR & GA-MR \& PSO-MR \\
$\mathbf{0 1 1}$ & 69.33 & 1589 & 48.82 & 1576 & GA-MR & GA-MR \\
$\mathbf{0 1 2}$ & 39.62 & 1098 & 17.92 & 1082 & GA-MR & GA-MR \\
$\mathbf{0 1 3}$ & 22.20 & 1290 & 13.97 & 1290 & GA-MR & GA-MR \& PSO-MR \\
$\mathbf{0 1 4}$ & 87.09 & 1404 & 32.94 & 1404 & GA-MR & GA-MR \& PSO-MR \\
$\mathbf{0 1 5}$ & 22.55 & 1168 & 31.78 & 1168 & PSO-MR & GA-MR \& PSO-MR \\
$\mathbf{0 1 6}$ & 10.21 & 1380 & 6.70 & 1380 & GA-MR & GA-MR \& PSO-MR \\
$\mathbf{0 1 7}$ & 12.17 & 1556 & 14.70 & 1556 & PSO-MR & GA-MR \& PSO-MR \\
$\mathbf{0 1 8}$ & 39.80 & 1199 & 23.84 & 1191 & GA-MR & GA-MR \\
$\mathbf{0 1 9}$ & 24.03 & 1518 & 8.17 & 1518 & GA-MR & GA-MR \& PSO-MR \\
$\mathbf{0 2 0}$ & 10.48 & 1581 & 6.80 & 1581 & GA-MR & GA-MR \& PSO-MR \\
$\mathbf{A V E}$ & $\mathbf{2 5 . 8 5}$ & $\mathbf{1 4 4 9 . 4 5}$ & $\mathbf{1 7 . 5 1}$ & $\mathbf{1 4 4 7 . 6}$ & GA-MR & GA-MR \\
\hline & & & & & &
\end{tabular}

MR, by saving $776.095 \mathrm{~s}$ and $786.125 \mathrm{~s}$ compared to the average time consumed by GAMS software, can generate optimal solutions, PSO-MR and GA-MR have relatively good performance in terms of the time spent for modeling and solving by GAMS.

\subsection{Comparing PSO-MR with GA-MR}

Given that modeling and problem solving by GAMS optimization are only appropriate for very small problems and cannot be justified for larger problems due to the required long time, the meta-heuristic algorithms can be used to generate near optimal solutions in short time. Therefore, in this paper, two improved metaheuristic algorithms, known as PSO-MR and GA-MR, are presented to tackle this problem. In order to evaluate the performance of PSO-MR and GA-MR for large-size problems, the number of 50 test problems in different scales are designed and their results are shown in Table 4. The designed problems are also solved separately with the presented PSO-MR and the 
results are registered in terms of quality and time spent on obtaining the solutions. The results are compared with those of the presented GA-MR.

For the problems with IDs from 001 to 020 , according to the results presented in Table 4 and Figure 9 , it can be observed that $85 \%$ of the solutions obtained by the PSO-MR are equal to best solutions achieved by problem solving through GA-MR and the results for merely $15 \%$ of the cases are worse. The solutions obtained by the proposed PSO-MR and GAMR algorithms are 1449.45 and 1447.6, respectively, on average. Thus, it can be concluded that the two improved algorithms can generate solutions with nearly identical quality for very-small-scale problems.

On the other hand, as shown in Table 4 and Figure 10, the required times for achieving solutions by PSO-MR and GA-MR algorithms are on average $25.88 \mathrm{~s}$ and $17.51 \mathrm{~s}$, respectively. It can be argued that in very-small-scale problems, the GA-MR has better performance than PSO-MR in terms of time.

For small-scale problems, by observing the results in Table 5 and Figure 11, it can be deduced that the solutions obtained by the PSO-MR are on average $1.4 \%$ worse than the best solutions obtained by problem solving through the GA-MR presented in this paper, while GA-MR saves time by $58.72 \%$. The solutions obtained by the proposed PSO-MR and GA-MR algorithms are on average 2131.6 and 2102.1, respectively. Therefore, it can be stated that the quality of generation solutions by these two algorithms is approximately the same and the major difference is in the time of achieving the solutions.

As shown in Table 5 and Figure 12, the required times for achieving the solutions by PSO-MR and GA$\mathrm{MR}$ algorithms are on average $389.77 \mathrm{~s}$ and $160.86 \mathrm{~s}$, respectively. It can be argued that in small-size problems, the GA-MR is better than PSO-MR in terms of time.

For the medium problems (IDs 031 to 040), according to the results in Table 6 and Figures 13 and 14, PSO-MR obtains the average quality of 5766.4 in $2618.5 \mathrm{~s}$, while GA-MR achieves $3.14 \%$ better results on average by $965.7 \mathrm{~s}$ time saving. Thus, it can be stated that the quality of solutions generated by the two

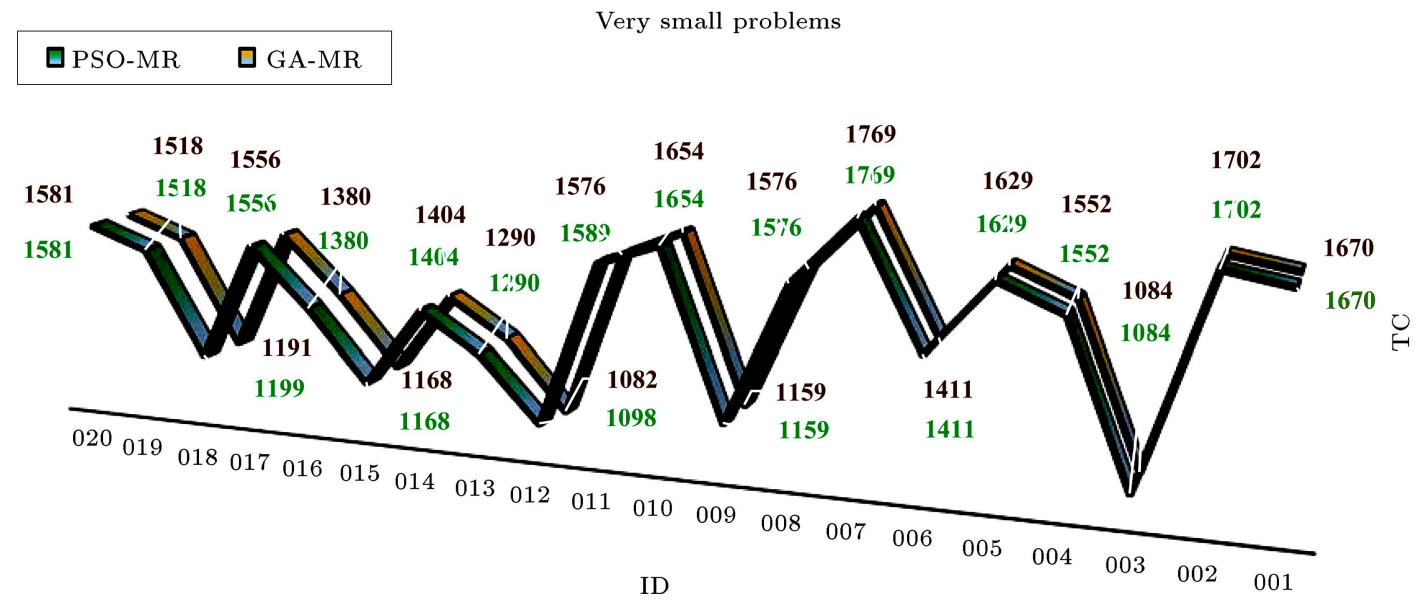

Figure 9. Results obtained through solving very small problems by Particle Swarm Optimization with Multiple Routes (PSO-MR) and Genetic Algorithm with Multiple Route (GA-MR) in terms of TC.

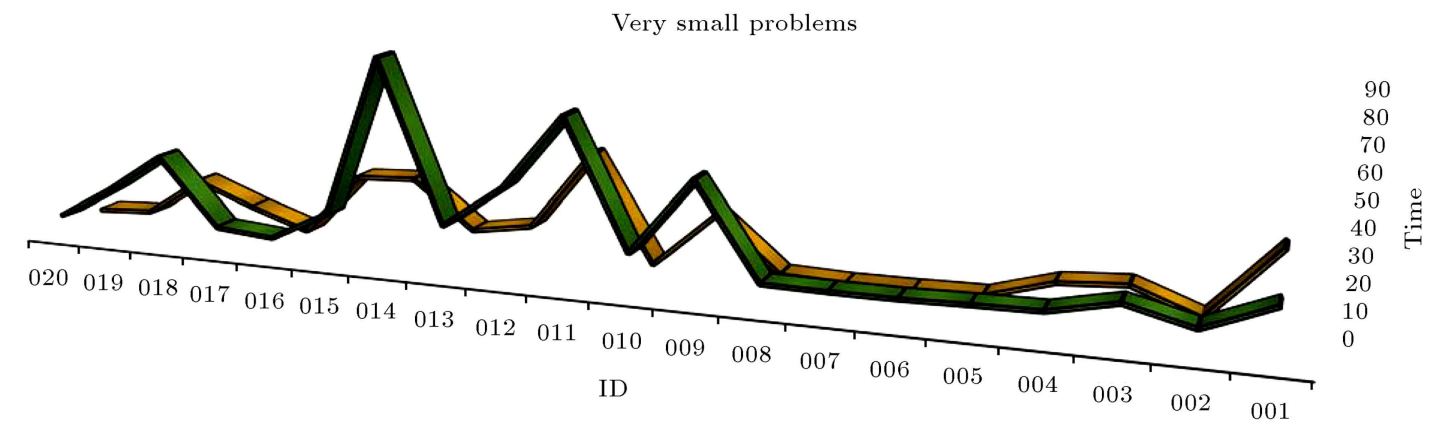

\begin{tabular}{|c|c|c|c|c|c|c|c|c|c|c|c|c|c|c|c|c|c|c|c|c|}
\hline & 020 & 019 & 018 & 017 & 016 & 015 & 014 & 013 & 012 & 011 & 010 & 009 & 008 & 007 & 006 & 005 & 004 & 003 & 002 & 001 \\
\hline 口PSO-MR & 10.48 & 24.03 & 39.8 & 12.17 & 10.21 & 22.55 & 87.09 & 22.2 & 39.62 & 69.33 & 18.51 & 49.08 & 11.8 & 11.47 & 11.48 & 12.08 & 12.52 & 18.21 & 11.86 & 22.62 \\
\hline 口GA-MR & 6.8 & 8.17 & 23.84 & 14.7 & 6.7 & 31.78 & 32.94 & 13.97 & 17.92 & 48.82 & 7.23 & 29.28 & 8.29 & 7.46 & 7.6 & 8.05 & 15.63 & 17.35 & 7.87 & 35.85 \\
\hline
\end{tabular}

Figure 10. Results obtained through solving very small problems by Particle Swarm Optimization with Multiple Routes (PSO-MR) and Genetic Algorithm with Multiple Route (GA-MR) in terms of time. 
Table 5. Computational results (small problems).

\begin{tabular}{ccccccc}
\hline \multicolumn{7}{c}{ Small sized problems } \\
\hline ID & \multicolumn{2}{c}{ PSO-MR } & \multicolumn{2}{c}{ GA-MR } & \multicolumn{2}{c}{ Best solution } \\
\cline { 2 - 7 } $\mathbf{0 2 1}$ & 252.97 & 1827 & 135.17 & 1804 & GA-MR & GA-MR \\
$\mathbf{0 2 2}$ & 574.31 & 2824 & 244 & 2775 & GA-MR & GA-MR \\
$\mathbf{0 2 3}$ & 161.65 & 1429 & 104.84 & 1411 & GA-MR & PSO-MR \\
$\mathbf{0 2 4}$ & 612.40 & 2728 & 139.79 & 2704 & GA-MR & GA-MR \\
$\mathbf{0 2 5}$ & 249.30 & 1949 & 280.87 & 1928 & PSO-MR & GA-MR \\
$\mathbf{0 2 6}$ & 109.27 & 1710 & 171.27 & 1692 & PSO-MR & GA-MR \\
$\mathbf{0 2 7}$ & 259.76 & 1996 & 64.57 & 1963 & GA-MR & GA-MR \\
$\mathbf{0 2 8}$ & 952.96 & 3038 & 301.16 & 2960 & GA-MR & GA-MR \\
$\mathbf{0 2 9}$ & 359.06 & 2136 & 75.61 & 2162 & GA-MR & PSO-MR \\
$\mathbf{0 3 0}$ & 366.05 & 1679 & 91.31 & 1622 & GA-MR & GA-MR \\
AVE & $\mathbf{3 8 9 . 7 7}$ & $\mathbf{2 1 3 1 . 6}$ & $\mathbf{1 6 0 . 8 6}$ & $\mathbf{2 1 0 2 . 1}$ & GA-MR & GA-MR \\
\hline
\end{tabular}

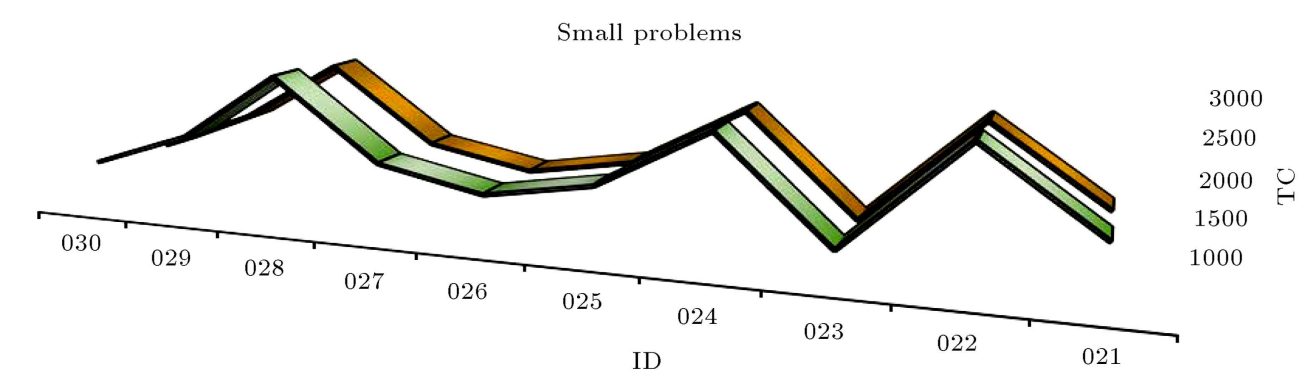

\begin{tabular}{|c|c|c|c|c|c|c|c|c|c|c|}
\hline & 030 & 029 & 028 & 027 & 026 & 025 & 024 & 023 & 022 & 021 \\
\hline 口PSO-MR & 1679 & 2136 & 3038 & 1996 & 1710 & 1949 & 2728 & 1429 & 2824 & 1827 \\
\hline 口GA-MR & 1622 & 2162 & 2960 & 1963 & 1692 & 1928 & 2704 & 1411 & 2775 & 1804 \\
\hline
\end{tabular}

Figure 11. Results obtained through solving small problems by Particle Swarm Optimization with Multiple Routes (PSO-MR) and Genetic Algorithm with Multiple Route (GA-MR) in terms of TC.

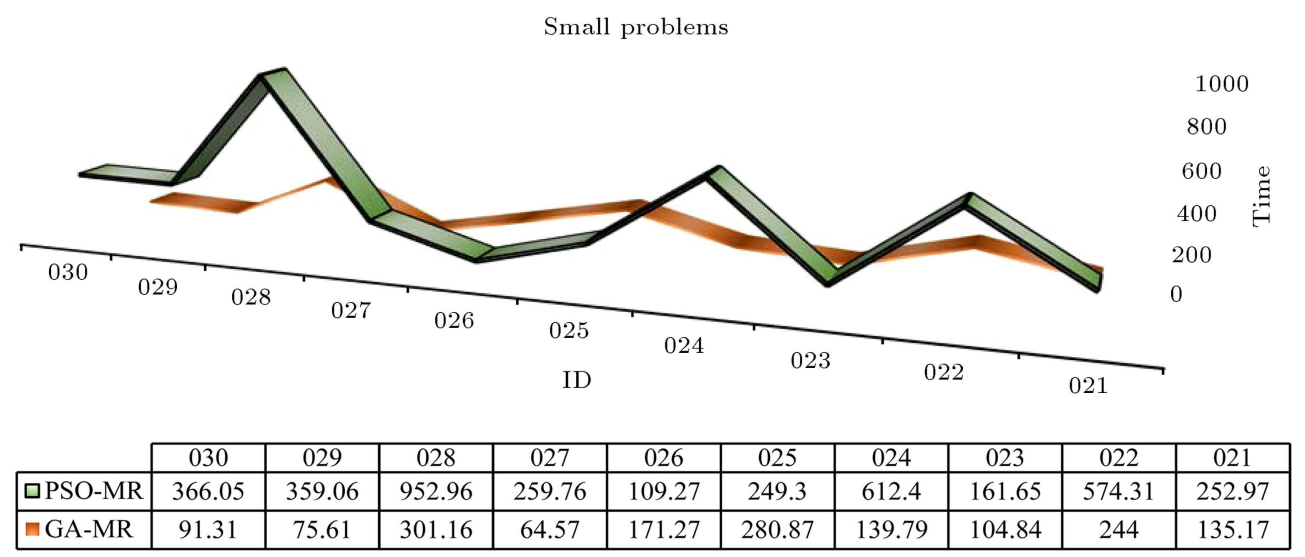

Figure 12. Results obtained through solving small problems by Particle Swarm Optimization with Multiple Routes (PSO-MR) and Genetic Algorithm with Multiple Route (GA-MR) in terms of time.

algorithms is almost the same and the main difference is in the time of achieving the solutions. The PSO$\mathrm{MR}$ algorithm requires a longer time than GA-MR for achieving solutions to the medium-size problems.

For the large-size problems (IDs 041 to 050), based on the results in Table 7 and Figures 15 and
16 , it can be stated that the solutions obtained by the proposed PSO-MR and GA-MR are on average 10401.2 and 9899.4, respectively. Also, the time spent on solving the problems is on average 7702.2 and 5048.9 by the PSO-MR and GA-MR, respectively. Considering the quality of solutions, it can be mentioned that 
Table 6. Computational results (medium problems).

\begin{tabular}{ccccccc}
\hline \multicolumn{7}{c}{ Medium sized problems } \\
\hline & \multicolumn{2}{c}{ PSO-MR } & \multicolumn{2}{c}{ GA-MR } & \multicolumn{2}{c}{ Best solution } \\
\cline { 2 - 7 } ID & Time (s) & TC & Time (s) & TC & Time (s) & TC \\
\hline $\mathbf{0 3 1}$ & 1975 & 5292 & 1087 & 5127 & GA-MR & GA-MR \\
$\mathbf{0 3 2}$ & 521.02 & 2416 & 261.45 & 2282 & GA-MR & GA-MR \\
$\mathbf{0 3 3}$ & 1259 & 4715 & 963 & 4655 & GA-MR & GA-MR \\
$\mathbf{0 3 4}$ & 4306 & 8610 & 2702 & 8304 & GA-MR & GA-MR \\
$\mathbf{0 3 5}$ & 3448 & 5785 & 2433 & 5556 & GA-MR & GA-MR \\
$\mathbf{0 3 6}$ & 1545 & 5399 & 718 & 5300 & GA-MR & GA-MR \\
$\mathbf{0 3 7}$ & 3682 & 7017 & 2459 & 6924 & GA-MR & GA-MR \\
$\mathbf{0 3 8}$ & 4165 & 6077 & 2614 & 5719 & GA-MR & GA-MR \\
$\mathbf{0 3 9}$ & 2578 & 6245 & 1532 & 6014 & GA-MR & GA-MR \\
$\mathbf{0 4 0}$ & 2706 & 6108 & 1759 & 5971 & GA-MR & GA-MR \\
$\mathbf{A V E}$ & $\mathbf{2 6 1 8 . 5}$ & $\mathbf{5 7 6 6 . 4}$ & $\mathbf{1 6 5 2 . 8}$ & $\mathbf{5 5 8 5 . 2}$ & GA-MR & GA-MR \\
\hline
\end{tabular}

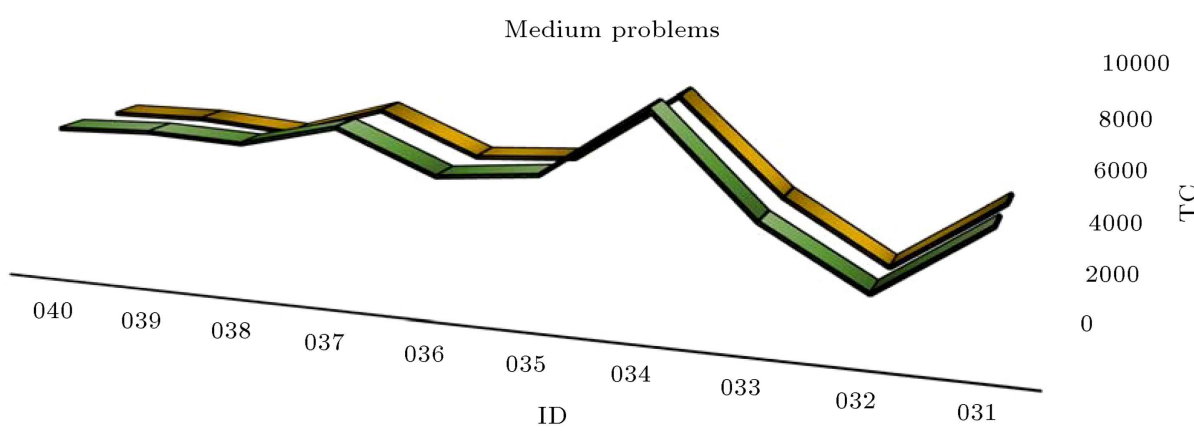

\begin{tabular}{|l|c|c|c|c|c|c|c|c|c|c|}
\cline { 2 - 10 } \multicolumn{1}{c|}{} & 040 & 039 & 038 & 037 & 036 & 035 & 034 & 033 & 032 & 031 \\
\hline$\square$ PSO-MR & 6108 & 6245 & 6077 & 7017 & 5399 & 5785 & 8610 & 4715 & 2416 & 5292 \\
\hline$\square$ GA-MR & 5971 & 6014 & 5719 & 6924 & 5300 & 5556 & 8304 & 4655 & 2282 & 5127 \\
\hline
\end{tabular}

Figure 13. Results obtained through solving medium problems by Particle Swarm Optimization with Multiple Routes (PSO-MR) and Genetic Algorithm with Multiple Route (GA-MR) in terms of TC.

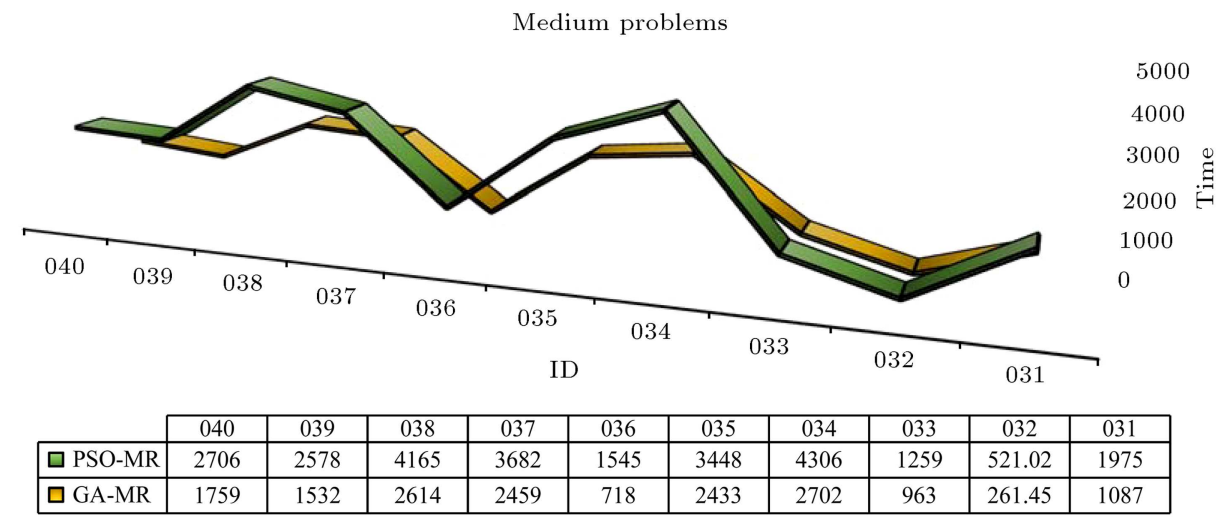

Figure 14. Results obtained through solving the medium problems by Particle Swarm Optimization with Multiple Routes (PSO-MR) and Genetic Algorithm with Multiple Route (GA-MR) in terms of time.

the GA-MR algorithm achieves the best solutions in all the cases and the PSO-MR algorithm generates close solutions. Thus, it can be said that in largesize problems, the quality of solutions generated by GA-MR is better than those by PSO-MR. Also, the PSO-MR algorithm requires longer time for achieving appropriate solutions to the problems than GA-MR algorithm does.

\section{Conclusions}

Regarding the significance of some technologies in 
Table 7. Computational results (large problems).

\begin{tabular}{ccccccc}
\hline \multicolumn{7}{c}{ Large sized problems } \\
\hline & \multicolumn{7}{c}{ PSO-MR } & \multicolumn{2}{c}{ GA-MR } & \multicolumn{2}{c}{ Best solution } \\
\cline { 2 - 7 } ID & Time (s) & TC & Time (s) & TC & Time (s) & TC \\
\hline $\mathbf{0 4 1}$ & 5692 & 7847 & 3771 & 7600 & GA-MR & GA-MR \\
$\mathbf{0 4 2}$ & 4838 & 10316 & 3253 & 9661 & GA-MR & GA-MR \\
$\mathbf{0 4 3}$ & 5246 & 8634 & 3180 & 8194 & GA-MR & GA-MR \\
$\mathbf{0 4 4}$ & 3280 & 8484 & 2098 & 7924 & GA-MR & GA-MR \\
$\mathbf{0 4 5}$ & 7955 & 13310 & 5117 & 12604 & GA-MR & GA-MR \\
$\mathbf{0 4 6}$ & 18445 & 16519 & 11611 & 15838 & GA-MR & GA-MR \\
$\mathbf{0 4 7}$ & 5281 & 7920 & 3638 & 7459 & GA-MR & GA-MR \\
$\mathbf{0 4 8}$ & 10292 & 11438 & 7109 & 11103 & GA-MR & GA-MR \\
$\mathbf{0 4 9}$ & 5646 & 7928 & 3763 & 7515 & GA-MR & GA-MR \\
$\mathbf{0 5 0}$ & 10347 & 11616 & 6949 & 11096 & GA-MR & GA-MR \\
$\mathbf{A V E}$ & $\mathbf{7 7 0 2 . 2}$ & $\mathbf{1 0 4 0 1 . 2}$ & $\mathbf{5 0 4 8 . 9}$ & $\mathbf{9 8 9 9 . 4}$ & GA-MR & GA-MR \\
\hline
\end{tabular}

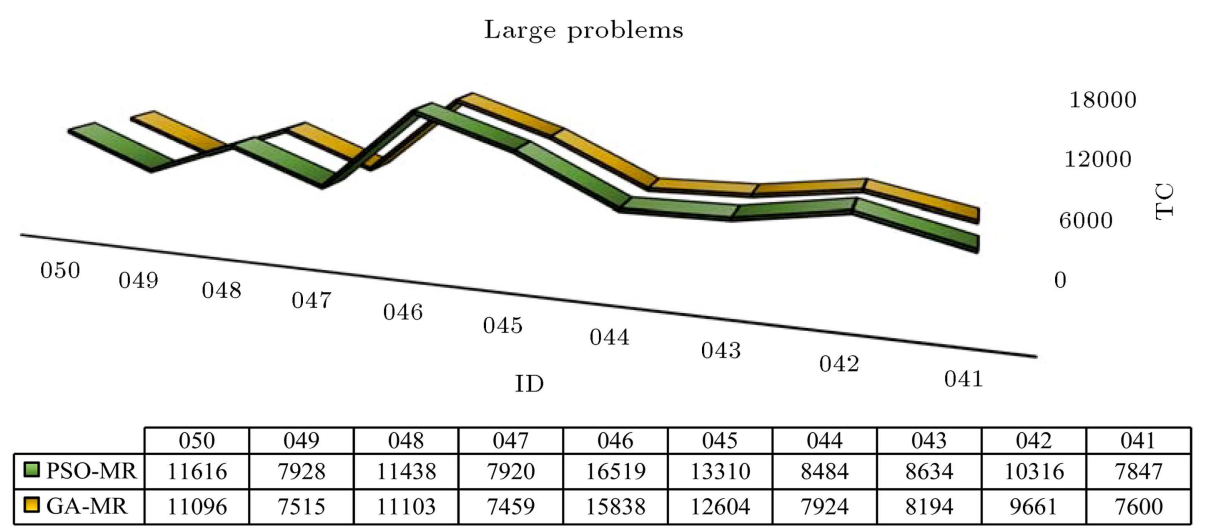

Figure 15. Results obtained through solving large problems by Particle Swarm Optimization with Multiple Routes (PSO-MR) and Genetic Algorithm with Multiple Route (GA-MR) in terms of TC.

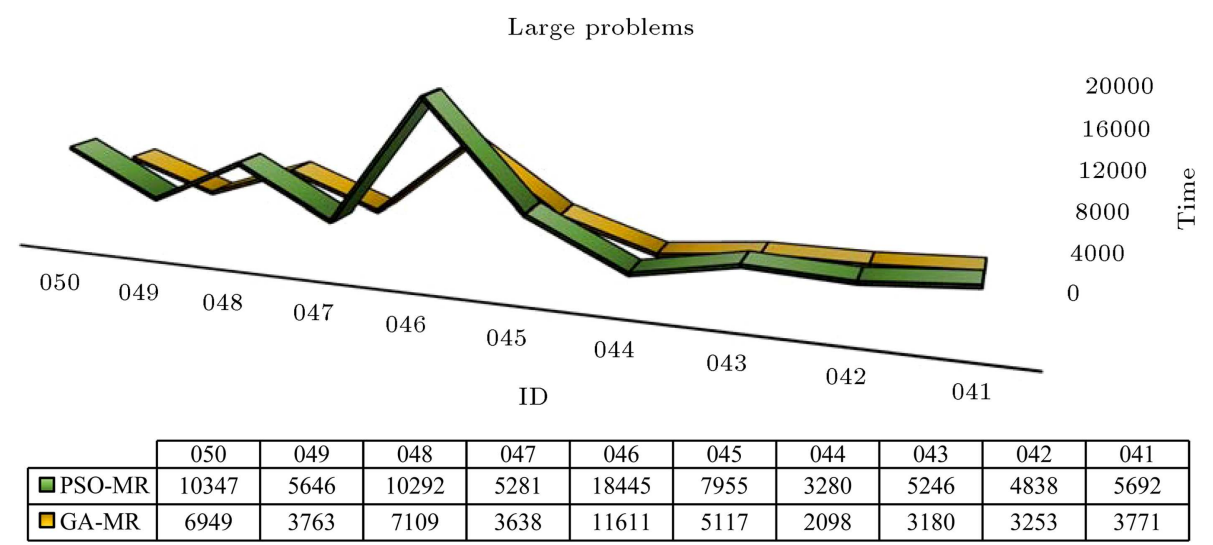

Figure 16. Results obtained through solving large problems by Particle Swarm Optimization with Multiple Routes (PSO-MR) and Genetic Algorithm with Multiple Route (GA-MR) in terms of time.

performing several parts of a project, they have not been properly examined in the Resource-Constrained Project Scheduling Problem (RCPSP) in the literature. Selecting the best technology is crucial with regard to changes in the main network of a project in terms of the number of activities required for the implementation, precedence relationships, allocation of resources, and consequently, the time and cost of project completion. Hence, in this paper, RCPSP with multiple routes for flexible project parts, namely Resource-Constrained Project Scheduling Problem with Multiple Routes (RCPSP-MR), was proposed with the aim of minimiz- 
ing the cost of project completion. The cost of project completion, in this paper, was divided into three parts: 1) the fixed cost of the availability of limited resources during the implementation of the project; 2) the cost of using limited resources with different implementation levels for each project activity; and 3) the cost of using non-renewable resources for implementing any project activity. In this paper, a new mathematical formulation of RCPSP-MR was firstly presented to find optimal solutions to the problem. Also, in order to solve large-size problems, two improved meta-heuristic algorithms, namely PSO-MR and GA-MR, were presented. First, in the proposed Genetic Algorithm (GA), due to the importance of producing initial solutions, each chromosome in the problem space was divided into seven parts, among which two were considered the sequencing the total project activities (such as fixed and flexible activities); one was related to the route and selected for flexible parts; and finally four parts were considered for the assigned reassurances and the level of quality for implementation activities. Solutions were frequently reinforced by crossover and mutation operations and in each iteration, a specification of high quality solutions was saved. Second, since the solution space presented for the problem in this paper was discrete and the proposed Particle Swarm Optimization (PSO) algorithm was used in continuous space, it was necessary to use an appropriate method to transform the production solutions into the continuous space. The method used in this paper was as follows: for each number of project activities, an equal interval between 0 to 1 was considered. Selection of the numbers inside each interval in each iteration indicated the selection of the corresponding activity. Also, in the proposed algorithm, in order to produce quality solutions, mutation was used for the GA algorithm and the initial solution was presented. Based on the velocity vector in each iteration, the obtained solutions by the proposed algorithm were moved towards the optimal solution. In order to assess the performance of the mathematical model and the proposed algorithms, the number of 50 test problems (in very small, small, medium, and large sizes) were designed and solved using the mathematical model and the proposed algorithms. Then, the results were compared. The computational results showed that the proposed GA-MR generated highquality solutions compared to the PSO-MR in a timely fashion. In very small problems, the results obtained by PSO-MR, GA-MR, and the mathematical model were approximately the same. Therefore, it can be concluded that the proposed methods have decent performance in terms of solution quality. In small and medium problems, it was observed that PSO-MR and GA-MR had relatively appropriate performance from the same perspective. However, GA-MR could obtain the results in less time than PSO-MR did. Finally, in large-size problems, the quality of solutions generated by GA-MR was better than that by PSO-MR. Also, the PSO-MR algorithm required longer time for achieving appropriate solutions to the large-size problems than GA-MR algorithm did. The RCPSP-MR is an interesting method with high research potential. In this paper, a mixed-integer nonlinear programming model and two meta-heuristic algorithms (i.e., PSO$\mathrm{MR}$ and GA-MR) were proposed to solve the problems in hand with the objective of minimizing the total cost of project completion. In future researches, it is suggested to incorporate other well-known metaheuristic algorithms, (e.g., SA, TS, and ACO) with other economic objectives such as maximization of net present value or minimization of ordering costs during project implementation. Moreover, it can be resource-based and deal with the resource investment problem. In addition, the problem can be extended to encompass real world problems. To this aim, another recommendation can be the use of concepts like preemptive scheduling, setup time, time lag, release date, and deadline.

\section{Acknowledgements}

The authors are thankful to the editor and anonymous reviewers for their constructive comments and recommendations, which remarkably improved the present paper.

\section{References}

1. Chand, S., Huynh, Q., Singh, H., Ray, T., and Wagner, M. "On the use of genetic programming to evolve priority rules for resource constrained project scheduling problem", Information Sciences, 432, pp. 146-163 (2018).

2. Dorfeshan, Y., Mousavi, S.M., Vahdani, B., and Siadat, A. "Determining project characteristics and critical path by a new approach based on modified NWRT method and risk assessment under an interval type-2 fuzzy environment", Scientia Iranica, 26(4), pp. 2579-2600 (2019).

3. Dorfeshan, Y., Mousavi, S.M., Mohagheghi, V., and Vahdani, B. "Selecting project-critical path by a new interval type-2 fuzzy decision methodology based on MULTIMOORA, MOOSRA and TPOP methods", Computers \& Industrial Engineering, 120, pp. 160178 (2018).

4. Dorfeshan Y. and Mousavi, S.M. "A new interval type2 fuzzy decision method with an extended relative preference relation and entropy to project critical path selection", International Journal of Fuzzy System Applications, 8(1), pp. 19-47 (2019).

5. Mohagheghi, V., Mousavi, S.M., and Vahdani, B. "Analyzing project cash flow by a new interval type2 fuzzy model with an application to construction 
industry", Neural Computing and Applications, 28, pp. 3393-3411 (2017).

6. Mohagheghi V., Mousavi, S.M., Vahdani, B., and Siadat, A. "A mathematical modeling approach for high and new technology-project portfolio selection under uncertain environments", Journal of Intelligent and Fuzzy Systems, 32, pp. 4069-4079 (2017).

7. Moradi, N., Mousavi, S.M., and Vahdani, B. "An earned value model with risk analysis for project management under uncertain conditions", Journal of Intelligent and Fuzzy Systems, 32, pp. 97-113 (2017).

8. Moradi, N., Mousavi, S.M., and Vahdani, B. "An interval type-2 fuzzy model for project-earned value analysis under uncertainty", Journal of Multiple-Valued Logic and Soft Computing, 30, pp. 79-103 (2018).

9. Vanhoucke, M., Project Management with Dynamic Scheduling, Springer, Berlin, Heidelberg, online ISBN: 978-3-642-25175-7 (2013).

10. Hartmann, D. and Briskorn, A. "Survey of variants and extensions of the resource constrained projectscheduling problem", European Journal of Operational Research, 207(1), pp. 1-14 (2010).

11. Okubo, H., Miyamoto, T., Yoshida, S., Mori, K., Kitamura, S., and Izui, Y. "Project scheduling under partially renewable resources and resource consumption during setup operations", Computers and Industrial Engineering, 83, pp. 91-99 (2015).

12. Szeredi, R. and Schutt, A. "Modeling and solving multi-mode resource-constrained project scheduling", International Conference on Principles and Practice of Constraint, 9892, pp. 483-492 (2016).

13. Habibi, F., Barzinpour, F., and Sadjadi, S.J. "Resource-constrained project scheduling problem: review of past and recent developments", Journal of Project Management, 3(2), pp. 55-88 (2018).

14. Blazewicz, J., Lenstra, J., and Rinnooy Kan, A. "Scheduling subject to resource constraints: classification and complexity", Discrete Applied Mathematics, 5(1), pp. 11-24 (1983).

15. Zhu, J., Li, X., and Shen, W. "Effective genetic algorithm for resource-constrained project scheduling with limited preemptions", International Journal of Machine Learning and Cybernetics, 2(2), pp. 55-65 (2011).

16. Agarwal, A., Colak, S., and Erenguc, S. "A neurogenetic approach for the resource-constrained project scheduling problem", Computers and Operations Research, 38(1), pp. 44-50 (2011).

17. Fang, C. and Wang, L. "An effective shuffled frogleaping algorithm for resource-constrained project scheduling problem", Computers and Operations Research, 39(5), pp. 890-901 (2012).

18. Zamani, R. "A competitive magnet-based genetic algorithm for solving the resource-constrained project scheduling problem", European Journal of Operational Research, 229(2), pp. 552-559 (2013).
19. Zeighami, V., Akbari, R., and Ziarati, K. "Development of a method based on particle swarm optimization to solve resource constrained project scheduling problem", Scientia Iranica, 20(6), pp. 2123-2137 (2013).

20. Sahli, A., Carlier, J., and Moukrim, A. "Comparison of mixed integer linear programming models for the event scheduling problem with consumption and production of resources", IFAC-PapersOnLine, 49(12), pp. 10441049 (2016).

21. Xiao, J., Wu, Z., Hong, X.X., Tang, J.C., and Tang, Y. "Integration of electromagnetism with multi-objective evolutionary algorithms for RCPSP", European Journal of Operational Research, 251(1), pp. 22-35 (2016).

22. Vanhoucke, M. and Coelho, J. "An approach using SAT solvers for the RCPSP with logical constraints", European Journal of Operational Research, 249(2), pp. 577-591 (2016).

23. Bibiks, K., Hu, F., Li, J.P., and Smith, A. "Discrete cuckoo search for resource-constrained project scheduling problem", IEEE 18th International Conference on Computational Science and Engineering (2015). DOI: 10.1109/CSE.2015.39

24. Bibiks, K., Hu, Y.F., Li, J.P., Pillai, P., and Smith, A. "Improved discrete cuckoo search for the resourceconstrained project scheduling problem", Applied Soft Computing, 69, pp. 493-503 (2018).

25. Fathallahi, F. and Najafi, A. "A hybrid genetic algorithm to maximize net present value of project cash flows in resource-constrained project scheduling problem with fuzzy parameters", Scientia Iranica, 23(4), pp. 1893-1903 (2016).

26. Gonzalez-Pardo, A., Del Ser, J., and Camacho, D. "Comparative study of pheromone control heuristics in ACO algorithms for solving RCPSP problems", Applied Soft Computing, 60, pp. 241-255 (2017).

27. Kadri, R.L. and Boctor, F.F. "An efficient genetic algorithm to solve the resource-constrained project scheduling problem with transfer times: The single mode case", European Journal of Operational Research, 265(2), pp. 454-462 (2018).

28. Coelho, J. and Vanhoucke, M. "An exact composite lower bound strategy for the resource-constrained project scheduling problem", Computers and Operations Research, 93, pp. 135-150 (2018).

29. Chakrabortty, R.K., Sarker, R.A., and Essam, D.L. "Multi-mode resource constrained project scheduling under resource disruptions", Computers and Chemical Engineering, 88, pp. 13-29 (2016).

30. Kopanos, G.M., Kyriakidis, T.S., and Georgiadis, M.C. "New continuous-time and 2 discrete-time mathematical formulations for resource-constrained project scheduling problems", Computers and Chemical Engineering, 68, pp. 96-106 (2014). 
31. Peteghem, V.V. and Vanhoucke, M. "An experimental investigation of Meta heuristics for the multi-mode resource constrained project-scheduling problem on new dataset instances", European Journal of Operational Research, 235(1), pp. 62-72 (2014).

32. Besikci, U., Bilge, U., and Ulusoy, G. "Multi mode resource constrained multi-project scheduling and resource portfolio problem", European Journal of Operational Research, 240(1), pp. 22-31 (2015).

33. Fernandes Muritiba, A.E., Rodrigues, C.D., and Da Costa, F.A. "A path-relinking algorithm for the multimode resource-constrained project scheduling problem", Computers and Operations Research, 92, pp. 145-154 (2018).

34. Van Den Eeckhout, M., Maenhout, B., and Vanhoucke, M. "A heuristic procedure to solve the project staffing problem with discrete time/resource trade-offs and personnel scheduling constraints", Computers and Operations Research, 101, pp. 144-161 (2019).

35. Bellenguez-Morineau, O. and Neron, E. "A Branchand-bound method for solving multi-skill project scheduling problem", RAIRO Operations Research, 41(2), pp. 155-170 (2007).

36. Correia, I. and Saldanha-da-Gama, F. "The impact of fixed and variable costs in a multi-skill projectscheduling problem: An empirical study", Computers and Industrial Engineering, 72, pp. 230-238 (2014).

37. Correia, I. and Saldanha-da-Gama, F. "A modeling framework for project staffing and scheduling problems", In: Schwindt C., Zimmermann J., Eds., Handbook on Project Management and Scheduling, International Handbooks on Information Systems, Springer, Cham, 1, pp. 547-564 (2015).

38. Maghsoudlou, H., Afshar-Nadjafi, B., and Akhavan Niaki, S.T. "A multi-objective invasive weeds optimization algorithm for solving multi-skill multimode resource constrained project scheduling problem", Computers and Chemical Engineering, 88, pp. 157-169 (2016).

39. Javanmard, S., Afshar Nadjafi, B., and Akhavan Niaki, S.T. "Preemptive multi-skilled resource investment project scheduling problem: Mathematical modelling and solution approaches", Computers and Chemical Engineering, 96, pp. 55-68 (2017).

40. Wang, L. and Zheng, X.L. "A knowledge-guided multiobjective fruit fly optimization algorithm for the multiskill resource constrained project scheduling problem", Swarm and Evolutionary Computation, 38, pp. 54-63 (2018).

41. Myszkowski, P., Olech, L., Laszczyk, M., and Skowroński, M. "Hybrid differential evolution and greedy algorithm (DEGR) for solving multi-skill resource-constrained project scheduling problem", Applied Soft Computing, 62, pp. 1-14 (2018).
42. Benjaafar, S., Talavage, J., and Ramakrishnan, R. "The effect of routing and machine flexibility on the performance of manufacturing systems", International Journal of Computer Integrated Manufacture, 8(4), pp. 265-276 (1995).

43. Chan, F.T.S. "The effects of routing flexibility on a flexible manufacturing system", International Journal of Computer Integrated Manufacturing, 14(5), pp. 431-445 (2001).

44. Yu, X. and Ram, B. "Bio-inspired scheduling for dynamic job shops with flexible routing and sequencedependent setups", International Journal of Production Research, 44(22), pp. 4793-4813 (2006).

45. Golmakani, H.R. and Birjandi, A.R. "A two-phase algorithm for multiple-route job shop scheduling problem subject to makespan", International Journal of Advanced Manufacturing Technology, 67(1-4), pp. 203-216 (2013).

46. Golmakani, H.R. and Birjandi, A.R. "Multiple route job shop scheduling using particle swarm optimisation approach", International Journal of Procurement Management, 7(2), pp. 119-144 (2014).

47. Golmakani, H.R., Mills, J.K., and Benhabib, B. "Deadlock-free scheduling and control of flexible manufacturing cells using Automata theory", IEEE Transactions Systems, 36, pp. 327-337 (2006).

48. Golmakani, H.R., Mills, J.K., and Benhabib, B. "Online scheduling and control of flexible manufacturing cells using Automata theory", International Journal of Computer Integrated Manufacture, 19(2), pp. 178-193 (2007).

49. Golmakani, H.R. and Namazi, A. "An artificial immune algorithm for multiple-route job shop scheduling problem", International Journal of Advanced Manufacturing Technology, 63, pp. 77-86 (2012).

50. Kellenbrink, C. and Helber, S. "Scheduling resourceconstrained projects with a flexible project structure", European Journal of Operational Research, 246(2), pp. 379-391 (2015).

51. Tao, S. and Dong, Z.S. "Multi-mode resource constrained project scheduling problem with alternative project structure", Computers and Industrial Engineering, 125, pp. 333-347 (2018).

52. Nonaka, Y., Erdos, G., Kis, T., Nakano, T., and Vancza, J. "Scheduling with alternative routings in CNC workshops", CIRP Annals Manufacture Technology, 61(1), pp. 449-454 (2012).

53. Ozguven, C., Ozbakır, L., and Yavuz, Y. "Mathematical models for job-shop scheduling problems with routing and process plan flexibility", Applied Mathematical Modelling, 34(1), pp. 1539-1548 (2010).

54. Rajabinasab, A. and Mansour, S. "Dynamic flexible job shop scheduling with alternative process plans: an agent-based approach", International Journal of Advanced Manufacture Technology, 54(9-12), pp. 10911107 (2011). 
55. Rossi, A. and Dini, G. "Flexible job-shop scheduling with routing flexibility and separable setup times using ant colony optimization method", Robotics and Computer-Integrated Manufacturing, 23(5), pp. 503516 (2007).

56. Roshanaei, V., Vahdani, B., Mousavi, S.M., Mousakhani, M., and Zhang, G. "CAD/CAM system selection: A multi-component hybrid fuzzy MCDM model", Arabian Journal for Science and Engineering, 38(9), pp. 2579-2594 (2013).

57. Tavakkoli-Moghaddam, R., Heydar, M., and Mousavi, S.M. "A hybrid genetic algorithm for a bi-objective scheduling problem in a flexible manufacturing cell", International Journal of Engineering, Transaction A: Basics, 23(3-4), pp. 235-252 (2010).

58. Sharma, D., Singh, V., and Sharma, C. "GA based scheduling of FMS using roulette wheel selection process", Proceedings of the International Conference on SocProS, AISC, 131, pp. 931-940 (2011).

59. Holland, J.H. and Koza, J.R., Genetic Programming: On the Programming of Computers by Means of Natural Selection, MIT Press, ISBN: 0-262-61084-1 (1998).

60. Yassine, A.A., Mostafa, O., and Browning, T.R. "Scheduling multiple, resource-constrained, iterative, product development projects with genetic algorithms", Computers and Industrial Engineering, 107, pp. 39-56 (2017).

61. Zhang, H., Xie, J., Ge, J., Zhang, Z., and Zong, B. "A hybrid adaptively genetic algorithm for task scheduling problem in the phased array radar", European Journal of Operational Research, 272(3), pp. 868-878 (2019).

62. Kennedy, J. and Eberhart, R. "Particle swarm optimization", Proceedings of IEEE International Conference on Neural Networks, 4, pp. 1942-1948 (1995).

63. Mousavi, S.M., Vahdani, B., and Abdollahzade, M. "An intelligent model for cost prediction in new product development projects", Journal of Intelligent and Fuzzy Systems, 29(5), pp. 2047-2057 (2015).

64. Bewoor, L.A., Prakash, V.C., and Sapkal, S.U. "Production scheduling optimization in foundry using hybrid particle swarm optimization algorithm", Procedia Manufacturing, 22, pp. 57-66 (2018).

65. Joy, J., Rajeev, S., and Narayanan, V. "Particle swarm optimization for resource constrained-project scheduling problem with varying resource levels", Procedia Technology, 25, pp. 948-954 (2016).
66. Poli, R., Kennedy, J., and Blackwell, T. "Particle swarm optimization: an overview", Swarm Intelligence Journal, 1(1), pp. 33-57 (2007).

67. Li, J.Q., Pan, Q.K., Xie, S.X., Jia, B.X., and Wang, Y.T. "A hybrid particle swarm optimization and tabu search algorithm for flexible job-shop scheduling problem", The International Journal of Computer Theory and Engineering, 2(2), pp. 189-194 (2010).

\section{Biographies}

Alireza Birjandi received his BSc and MSc in Industrial Engineering from Tafresh University, Tafresh, Iran, in 2010 and 2013, respectively. $\mathrm{He}$ is a PhD candidate in Industrial Engineering at Islamic Azad University, South Tehran Branch, Tehran, Iran. His research interest is scheduling as well as project management and control. He has published several papers in international journals and conference proceedings.

Seyed Meysam Mousavi is an Associate Professor in the Department of Industrial Engineering, Faculty of Engineering, Shahed University in Tehran, Iran. He received a $\mathrm{PhD}$ degree from the School of Industrial Engineering at University of Tehran, Iran, and is currently a member of Iran's National Elite Foundation. He is now the Head of Industrial Engineering Department at Shahed University and a member of the Iranian Operational Research Association. His main research interests include cross-docking systems planning, logistics planning and scheduling, quantitative methods in project management, multiple criteria decision making under uncertainty, and applied soft computing. He has published many papers and book chapters in reputable journals and international conference proceedings.

Behnam Vahdani is an Assistant Professor in the Faculty of Industrial and Mechanical Engineering, Qazvin Branch, Islamic Azad University in Iran, and is a member of Iran's National Elite Foundation. His current research interests include supply chain network design, facility location and design, multi-criteria decision making, uncertain programming, meta-heuristics algorithms, and operations research applications. He has published numerous papers and book chapters in the aforementioned areas. 